University of Louisville

ThinkIR: The University of Louisville's Institutional Repository

Electronic Theses and Dissertations

8-2015

\title{
Order automorphisms on the lattice of residuated maps of some special nondistributive lattices.
}

\author{
Erika D. Foreman \\ University of Louisville
}

Follow this and additional works at: https://ir.library.louisville.edu/etd

Part of the Applied Mathematics Commons

\section{Recommended Citation}

Foreman, Erika D., "Order automorphisms on the lattice of residuated maps of some special nondistributive lattices." (2015). Electronic Theses and Dissertations. Paper 2257.

https://doi.org/10.18297/etd/2257

This Doctoral Dissertation is brought to you for free and open access by ThinkIR: The University of Louisville's Institutional Repository. It has been accepted for inclusion in Electronic Theses and Dissertations by an authorized administrator of ThinkIR: The University of Louisville's Institutional Repository. This title appears here courtesy of the author, who has retained all other copyrights. For more information, please contact thinkir@louisville.edu. 


\title{
ORDER AUTOMORPHISMS ON THE LATTICE OF RESIDUATED MAPS OF SOME SPECIAL NONDISTRIBUTIVE LATTICES
}

\author{
By \\ Erika D. Foreman \\ B.S., Georgetown College, 2009 \\ M.A., University of Louisville, 2011 \\ A Dissertation \\ Submitted to the Faculty of the \\ College of Arts and Sciences of the University of Louisville \\ in Partial Fulfillment of the Requirements \\ for the Degree of \\ Doctor of Philosophy \\ in \\ Applied and Industrial Mathematics \\ Department of Mathematics \\ University of Louisville \\ Louisville, KY
}

August 2015 



\title{
ORDER AUTOMORPHISMS ON THE LATTICE OF RESIDUATED MAPS OF SOME SPECIAL NONDISTRIBUTIVE LATTICES
}

\author{
Submitted by \\ Erika D. Foreman
}

A Dissertation Approved on

May 21, 2015

by the Following Reading and Examination Committee:

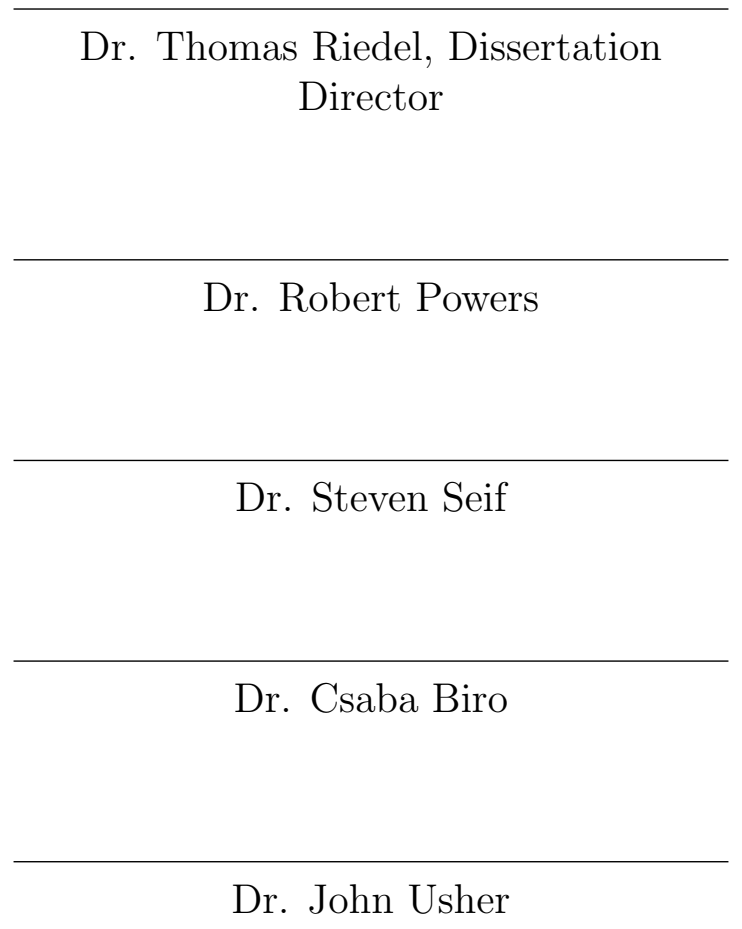




\section{DEDICATION}

To my mom. 


\section{ACKNOWLEDGEMENTS}

I would like to thank my advisor, Dr. Thomas Riedel, for his continued support in my research and professional pursuits. I would also like to thank the members of my dissertation committee, Dr. Robert Powers, Dr. Steven Seif, Dr. Csaba Biro, and Dr. John Usher, for their time and interest. Finally, thank you to my fellow graduate students who suffered with me, studied with me, and loved mathematics with me. 


\section{ABSTRACT \\ ORDER AUTOMORPHISMS ON THE LATTICE OF RESIDUATED MAPS OF SOME SPECIAL NONDISTRIBUTIVE LATTICES

\author{
Erika D. Foreman
}

May 21, 2015

The residuated maps from a lattice $L$ to itself form their own lattice, which we denote $\operatorname{Res}(L)$. In this dissertation, we explore the order automorphisms on the lattice $\operatorname{Res}(L)$ where $L$ is a finite nondistributive lattice. It is known that left and right composition of $f \in \operatorname{Res}(L)$ with automorphisms of $L$ yields an order automorphism of $\operatorname{Res}(L)$. It begs the question, then, if all order automorphisms of $\operatorname{Res}(L)$ can be classified as such. 


\section{TABLE OF CONTENTS}

DEDICATION ............................. iii

ACKNOWLEDGEMENTS . . . . . . . . . . . . . iv

ABSTRACT ........................ $\mathrm{v}$

LIST OF TABLES . . . . . . . . . . . . . . . . vii v vi

LIST OF FIGURES ....................... viii

1. INTRODUCTION .......................... 1

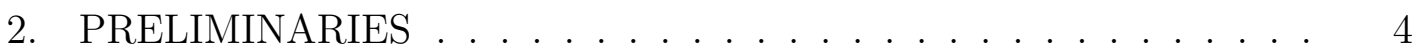

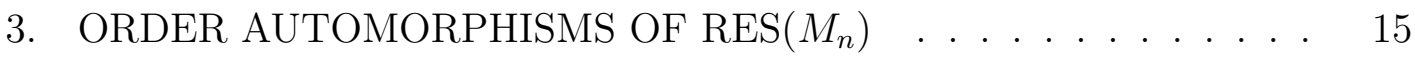

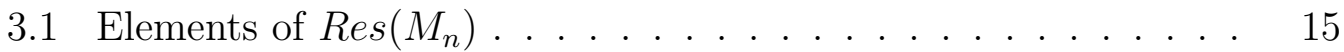

3.2 Join-Irreducibles of $\operatorname{Res}\left(M_{n}\right)$. . . . . . . . . . . . . . . . 19

3.3 Order Automorphisms of Res $\left(M_{2}\right)$. . . . . . . . . . . . . . 22

3.4 Order Automorphisms of $\operatorname{Res}\left(M_{n}\right)$ for $n \geq 3 \ldots . . . . . .23$

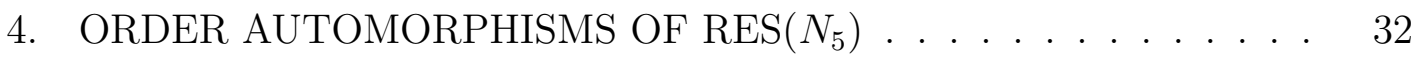

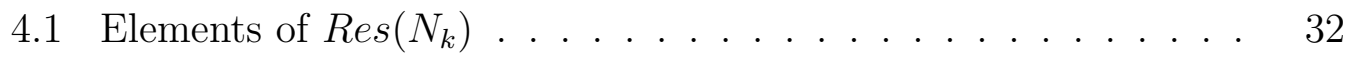

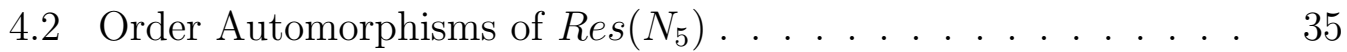

5. ORDER AUTOMORPHISMS OF RES $\left(M_{3} \times 2\right) \ldots . . . . . . \quad 40$

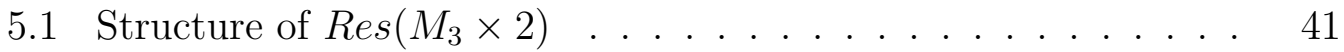

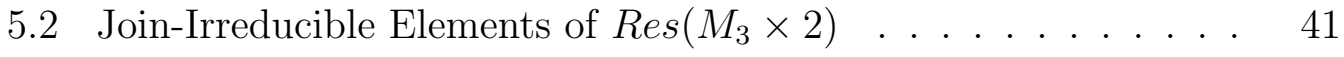

5.3 Order Automorphisms of $\operatorname{Res}\left(M_{3} \times 2\right)$, with an assumption . . 46

6. REMARKS AND CONCLUSION ............... 55

REFERENCES . . . . . . . . . . . . . . . . . 57

CURRICULUM VITAE . . . . . . . . . . . . . . . 60 


\section{LIST OF TABLES}

Table 3.1. Size of $\operatorname{Res}\left(M_{n}\right) \ldots \ldots \ldots$. . . . . . . . . . . . . . . . 18

Table 3.2. Join-Irreducibles of $\operatorname{Res}\left(M_{n}\right) \ldots \ldots \ldots$

Table 4.1. Size of $\operatorname{Res}\left(N_{k}\right) \ldots \ldots \ldots$. . . . . . . . . . 36

Table 5.1. $\operatorname{Res}\left(M_{3} \times 2\right) \quad \ldots \ldots \ldots \ldots$. . . . . . . . . . . 42 


\section{LIST OF FIGURES}

Figure 2.1. $M_{n} \ldots \ldots \ldots \ldots$

Figure $2.2 . \quad N_{k} \ldots \ldots \ldots \ldots \ldots$

Figure 3.1. $\operatorname{Res}\left(M_{2}\right) \ldots \ldots \ldots \ldots \ldots$

Figure 3.2. $\operatorname{Res}\left(M_{3}\right) \ldots \ldots \ldots \ldots \ldots$

Figure 3.3. $\quad$ Partial Structure of $\operatorname{Res}\left(M_{3}\right) \ldots \ldots \ldots$. . . . . . . . . . 21

Figure 3.4. Partial Structure of $\operatorname{Res}\left(M_{4}\right) \ldots \ldots$. . . . . . . . . . . . . 21

Figure 4.1. $N_{k} \ldots \ldots \ldots \ldots$. . . . . . . . . . . . . . . 32

Figure 4.2. Poset of Join-Irreducibles of $\operatorname{Res}\left(N_{5}\right) \quad \ldots$. . . . . . . . . . . 37

Figure 4.3. Covering relationship of $\delta_{b_{2}, 1}$ and $\delta_{0, b_{1}} \ldots \ldots$. . . . . 38

Figure 5.1. $M_{3} \times 2 \ldots \ldots \ldots \ldots$. . . . . . . . . . . 40 


\section{CHAPTER 1 INTRODUCTION}

The purpose of this dissertation is to investigate the order automorphisms on the lattice of residuated maps between lattices $L$ and $M$ (denoted $\operatorname{Res}(L, M)$ ), or more specifically on the lattice of residuated maps between $L$ and itself (denoted $\operatorname{Res}(L))$. Those familiar with Galois connections are in turn familiar with residuated maps, as a residuated-residual pair forms a Galois connection in the order-preserving definition of such. The definitions of residuated maps, their associated residual maps, and Galois connections can all be found in Chapter 2, but for now we will discuss their history and background in relation to this dissertation.

Galois connections of course go back to Évariste Galois, as they generalize the correspondence between subgroups and subfields explored in Galois theory. Birkhoff [2] and Raney [22] were some of the first to study Galois connections on lattices. Birkhoff studied constructing Galois connections on completely distributive lattices and Raney furthered his results by looking at Galois connections on complete lattices. Blyth and Janowitz [3] used these results on Galois connections to study the closely related residuated maps. They were interested in the study of semigroups, which motivated their research on residuated maps over Galois connections since the composition of two residuated maps is residuated, while the same is not true for Galois connections.

Shmuely [25] studied the algebraic properties of the lattice of residuated maps between two lattices $L$ and $M$, denoted $\operatorname{Res}(L, M)$. Some of her results included that $\operatorname{Res}(L, M)$ is complete if and only if $L$ and $M$ are complete and, 
likewise, $\operatorname{Res}(L, M)$ is completely distributive if and only if $L$ and $M$ are completely distributive. Schreiner [23] then studied what he called basic tight residuated maps and tight residuated maps, which are what we will refer to as $\delta$ s and a join of $\delta \mathrm{s}$ later. His work led to the result that with $L$ and $M$ distributive lattices, every element of $\operatorname{Res}(L, M)$ can be written as a join of these basic tight maps (or $\delta \mathrm{s}$ ). This is not true in the nondistributive case as some elements of $\operatorname{Res}\left(M_{3}\right)$, which we will discuss later, cannot be written as a join of basic tight maps (i.e. $\delta$ s).

Powers [20][21] was the first to study the order automorphisms of residuated maps, denoted Aut(Res), and even their order automorphisms, that is Aut(Aut(Res)). His investigation was motivated by the study of probability distribution functions, as residuated maps find many applications in probability (see e.g. [24]) and percentile clustering [15]. His result noted that order automorphisms of $\operatorname{Res}([p, q],[r, s])$, in other words the non-decreasing, left continuous mappings from one closed real interval to another, are characterized by left and right compositions of bijections on the real intervals. These maps he called induced residuated maps.

As Powers studied residuated maps on a totally ordered set, the next natural step was to study residuated maps on a partially ordered set. Such a study can be motivated by its applications to formal concept analysis [5], cluster analysis [13], data mining and analysis [4] [6], computer programming [19], and fuzzy systems [1]. To investigate this question further, Marshall Lagani [18] studied residuated maps on distributive lattices. If $L$ and $M$ are distributive lattices, the join-irreducibles of $\operatorname{Res}(L, M)$ consist only of Schreiner's basic tight maps, or what we will call $\delta \mathrm{s}$. This allowed Lagani to narrow his investigation of $\operatorname{Res}(L, M)$ to only the partially ordered set of join-irreducibles. It is known (see e.g. [2]) in the distributive case that $\operatorname{Res}(L, M) \cong 2^{J(\operatorname{Res}(L, M))}$, the non-decreasing maps from $J(\operatorname{Res}(L, M))$ into the 2-chain, and $J(\operatorname{Res}(L, M)) \cong M(L)^{\partial} \times J(M)$. Lagani relied heavily on the works of Duffus [7] [8] and Farley[10] [11] to prove some preliminary results in the 
distributive case. He was able to construct some examples in the distributive case where not all order automorphisms of $\operatorname{Res}(L)$ are induced. For instance, if $L$ is the power set on two elements under set inclusion, often called $M_{2}$ or $2 \times 2$, then $L$ is a distributive lattice for which not all elements of $\operatorname{Aut}(\operatorname{Res}(L))$ are induced. This can be shown with a simple counting argument; there are $6 !=24$ order automorphisms of $\operatorname{Res}\left(M_{2}\right)$ but only 8 of them can be induced.

My work is to extend Lagani's results to the nondistributive case. From Lagani's results, we expected to easily find an example of a nondistributive lattice $L$ for which $\operatorname{Aut}(\operatorname{Res}(L))$ is not entirely characterized by induced mappings. However, an example of such has been quite difficult to conceive in the nondistributive case since even the smallest example is quite large. Since $M_{3}$ and $N_{5}$ are canonical nondistributive lattices, we begin by studying $A u t\left(\operatorname{Res}\left(M_{3}\right)\right)$ and prove these order automorphisms are completely characterized by left-right composition. We then generalize this result to $\operatorname{Aut}\left(\operatorname{Res}\left(M_{n}\right)\right)$ with $n>3$ for completeness sake. Then we study $\operatorname{Aut}\left(\operatorname{Res}\left(N_{5}\right)\right)$, which is also completely characterized by left-right composition, but for which the results are much more difficult to generalize to $\operatorname{Aut}\left(\operatorname{Res}\left(N_{k}\right)\right)$. Looking for an example for which not all order automorphisms are induced, we began studying $\operatorname{Res}\left(M_{3} \times 2\right)$, but our results so far have been inconclusive.

In Chapter 2, we establish preliminary definitions, notation, and results used throughout the remaining discussion. In Chapter 3, we examine the structure of $\operatorname{Res}\left(M_{n}\right)$, discuss the $\operatorname{Res}\left(M_{2}\right)$ case, and prove that order automorphisms of $\operatorname{Res}\left(M_{n}\right)$ for $n \geq 3$ are all induced by left-right composition. In Chapter 4, we discuss the structure and elements of $\operatorname{Res}\left(N_{k}\right)$ and prove the order automorphisms of $\operatorname{Res}\left(N_{5}\right)$ are also induced. In Chapter 5, we discuss some preliminary results on the residuated maps of $M_{3} \times 2$. In Chapter 6 , we make some observations and provide potential avenues for further research. 


\section{CHAPTER 2 \\ PRELIMINARIES}

We will begin by defining some necessary terms and stating some well-known results, many of which can be found in references [3] and [5]. First, we define the basic structures of order theory, including partially ordered sets and lattices.

DEFINITION 2.1 (partial order; partially ordered set). Let $P$ be a set. A partial order on $P$ is a binary relation $\leq$ on $P$ that is reflexive, antisymmetric, and transitive. That is, for all $x, y, z$ in $P$,

(i) $x \leq x$,

(ii) $x \leq y$ and $y \leq x$ imply $x=y$,

(iii) $x \leq y$ and $y \leq z$ imply $x \leq z$.

$A$ set $P$ equipped with a partial order is called a partially ordered set, or poset for short.

A partially ordered set conceptualizes the idea of a certain arrangement or order in a set; it consists of a set together with a binary relation that tells us some elements precede others in the set. However, it is called a partial order because it is not necessary that every pair of elements is related in the order. Two elements $a$ and $b$ unrelated in the order are called non-comparable, denoted $a \mid b$. This is in contrast to a totally ordered set, for example the Real numbers, in which the law of trichotomy holds; that is, for any two real numbers $a$ and $b$, either $a<b, b<a$, or $a=b$. In certain partially ordered sets (and all finite ones), we can talk about an 
element immediately preceding another and we call this element "covered" by the larger element.

DEFINITION 2.2 (covering relation). Let $P$ be a partially ordered set and let $x, y \in$ $P$. Then $x$ is covered by $y$ (denoted $x \preccurlyeq y$ ) or analogously $y$ covers $x$ (denoted $y \succcurlyeq x$ ) if $x<y$ and there is no $z \in P$ with $x<z<y$.

A partially ordered set has a bottom element $0_{P}$ if for all $x \in P, 0_{P} \leq x$. It has a top element $1_{P}$ if for all $x \in P, 1_{P} \geq x$. From a partially ordered set, we can also construct its dual by reversing its binary relation; we can think of this as "turning upside down" the partially ordered set.

DEFINITION 2.3 (dual of a partially ordered set). Given any ordered set $P$, the dual of $P$ is an ordered set denoted $P^{\partial}$ such that $x \leq y$ in $P^{\partial}$ if and only if $y \leq x$ in $P$.

Partially ordered sets also have the notion of suprema and infima, commonly referred to as join and meet in the order theoretic setting.

DEFINITION 2.4 (join; meet). If $S$ is a subset of $P$, then the join of $S$, denoted $\bigvee S$, is the least upper bound of $S$, given it exists. The meet of $S$, denoted $\bigwedge S$, is the greatest lower bound of $S$, given it exists. If $S=\{a, b\}$, we write $a \vee b$ and $a \wedge b$.

The join and meet of any two elements in a partially ordered set need not exist. If they do exist for all pairs, we have a special partially ordered set called a lattice.

DEFINITION 2.5 (lattice; complete lattice). A partially ordered set $P$ is called a lattice if $x \vee y$ and $x \wedge y$ exist for all $x, y \in P$. A lattice $P$ is called a complete lattice if for all $S \subset P, \bigvee S$ and $\bigwedge S$ exist.

We will also want to discuss the classes of distributive and modular lattices, whose definitions are below. 
DEFINITION 2.6 (distributive lattice). A lattice $L$ is said to be distributive if it satisfies the distributive law: for all $a, b, c \in L, a \wedge(b \vee c)=(a \wedge b) \vee(a \wedge c)$.

DEFINITION 2.7 (modular lattice). A lattice $L$ is said to be modular if it satisfies the modular law: for all $a, b, c \in L$ with $a \geq c, a \wedge(b \vee c)=(a \wedge b) \vee c$.

Note that the distributive law has an equivalent dual law that distributes join over meet: a lattice $L$ is distributive if for all $a, b, c \in L, a \vee(b \wedge c)=(a \vee b) \wedge(a \vee c)$. The next result follows quickly from the definitions.

LEMMA 2.1. If a lattice $L$ is distributive, then it is modular.

Proof. Suppose $L$ is distributive and $a, b, c \in L$ with $a \geq c$. Then we have $a \wedge(b \vee c)=$ $(a \wedge b) \vee(a \wedge c)=(a \wedge b) \vee c$.

Now we establish a consistent definition of two lattices important to the rest of our discussion. The lattice $M_{n}$ has a bottom element 0 , a top element 1 , and an antichain of $n$ elements in-between, which we will denote by $a_{1}, a_{2}, \ldots, a_{n}$. The lattice $N_{k}$ has a bottom element 0 , a top element 1 , a chain of $k-3$ elements we will denote with $b_{1}, b_{2}, \ldots, b_{k-3}$, and then a single element denoted $a$ that is non-comparable to any element in the chain. We often draw partially ordered sets using Hasse Diagrams, in which a line segment is drawn upward from $x$ to $y$ if $y$ covers $x$. Hasse Diagrams of $M_{n}$ and $N_{k}$ can be found in Fig. 2.1 and Fig. 2.2. The special cases $M_{3}$ and $N_{5}$ are of particular interest in order theory because of Theorem 2.1, which characterizes distributive and modular lattices. We will first discuss an important definition and then the theorem, a proof of which you can find in [5].

DEFINITION 2.8 (sublattice). Let $L$ be a lattice and $\emptyset \neq M \subseteq L$. Then $M$ is a sublattice of $L$ if $a, b \in M$ implies $a \vee b \in M$ and $a \wedge b \in M$.

THEOREM 2.1 (The $M_{3}-N_{5}$ Theorem). Let $L$ be a lattice. Then 
(i) $L$ is nondistributive if and only if it contains a sublattice isomorphic to $M_{3}$ or $N_{5}$.

(ii) $L$ is nonmodular if and only if it contains a sublattice isomorphic to $N_{5}$.

This characterization is of particular importance to this dissertation because we aim to study results on nondistributive lattices, and so the natural place to start is on the lattices $M_{3}$ and $N_{5}$.

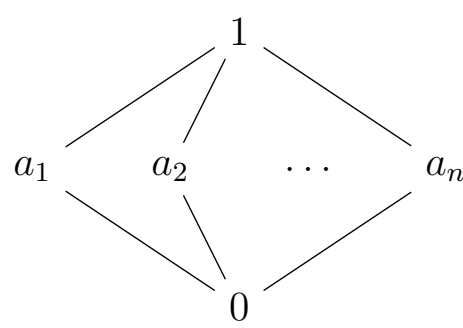

Figure 2.1: $M_{n}$

Nondistributive for $n \geq 3$

Modular for all $n$

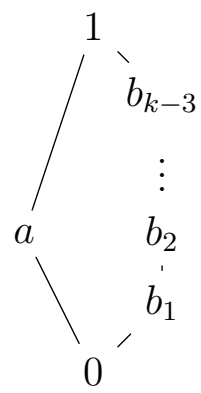

Figure 2.2: $N_{k}$

Nondistributive for $k \geq 5$

Nonmodular for $k \geq 5$

Our main result later will depend heavily on certain elements of a lattice called join-irreducibles and meet-irreducibles, which we define next. 
DEFINITION 2.9 (join-irreducible). Let $L$ be a lattice. An element $x \in L$ is joinirreducible if $x \neq 0_{L}$ and $x=a \vee b$ implies $x=a$ or $x=b$ for all $a, b \in L$.

DEFINITION 2.10 (meet-irreducible). Let $L$ be a lattice. An element $x \in L$ is meet-irreducible if $x \neq 1_{L}$ and $x=a \wedge b$ implies $x=a$ or $x=b$ for all $a, b \in L$.

Throughout this dissertation, we will often discuss maps between partially ordered sets and lattices. We will define some special types of maps below.

DEFINITION 2.11 (order-preserving; order-embedding; order-isomorphism). Let $P$ and $Q$ be partially ordered sets. $A$ map $f: P \rightarrow Q$ is said to be

(i) order-preserving if $x \leq y$ in $P$ implies $f(x) \leq f(y)$ in $Q$;

(ii) an order-embedding (denoted $f: P \hookrightarrow Q$ ) if $x \leq y$ in $P \Longleftrightarrow f(x) \leq f(y)$ in $Q$

(iii) an order-isomorphism if it is an order-embedding which maps $P$ onto $Q$.

DEFINITION 2.12 (lattice homomorphisms). Let $L$ and $M$ be lattices. A map $f: L \rightarrow M$ is said to be

(i) a join-homomorphism if for all $a, b \in L, f(a \vee b)=f(a) \vee f(b)$;

(ii) a complete join-homomorphism if for $A \subseteq P, f(\bigvee A)=\bigvee\{f(a) \mid a \in A\}$;

(iii) a meet-homomorphism if for all $a, b \in L, f(a \wedge b)=f(a) \wedge f(b)$;

(iv) a complete meet-homomorphism if for $A \subseteq P, f(\bigwedge A)=\bigwedge\{f(a) \mid a \in A\}$;

(v) a lattice homomorphism if $f$ is both a join-homomorphism and a meet-homomorphism.

This dissertation deals heavily with some very particular maps on partially ordered sets called residuated maps. The following definition of a residuated map can be found in [3]. In this context, $f$ is nondecreasing if $x<y$ in the domain implies $f(x) \leq f(y)$ in the range. 
DEFINITION 2.13 (residuated map). If $L, M$ are partially ordered sets, a map $f: L \rightarrow M$ is residuated if and only if $f$ is isotone (nondecreasing) and there exists a unique, isotone map $f^{+}: M \rightarrow L$ such that $f^{+} \circ f \geq i d_{L}$ and $f \circ f^{+} \leq i d_{M}$. The unique map $f^{+}$, called the residual of $f$, is defined such that $\forall y \in M, f^{+}(y)=$ $\max \{x \in L \mid f(x) \leq y\}$.

Those familiar with Galois connections can note that a residuated-residual pair forms a Galois connection in the order-preserving definition of such, found in texts such as the one by Davey and Priestley [5] in which they denote the residuated map by ${ }^{\triangleright}$ and its residual by $\triangleleft$. The order-preserving definition of a Galois connection appears often in the context of computer science; however, in mathematics, particularly in algebra, Galois connections are defined to be orderreversing. The difference, however, is not significant because we can switch between order-preserving and order-reversing by switching $M$ with $M^{\partial}$. The advantage of residuated maps over Galois connections is that the composition of two residuated maps is also residuated (as we see in Lemma 2.2), while the composition of two maps of a Galois connections is not necessarily part of a Galois connection.

LEMMA 2.2. If $f \in \operatorname{Res}(L, M)$ and $g \in \operatorname{Res}(M, P)$, then $g \circ f \in \operatorname{Res}(L, P)$ with $(g \circ f)^{+}=f^{+} \circ g^{+}$.

Proof. First, note that since $f, f^{+}, g$, and $g^{+}$are isotone, then so are $g \circ f$ and $f^{+} \circ g^{+}$. Next we prove that $f^{+} \circ g^{+}$is the special map $(g \circ f)^{+}$.

$$
\begin{aligned}
\left(f^{+} \circ g^{+}\right) \circ(g \circ f) & =f^{+} \circ\left(g^{+} \circ g\right) \circ f \\
& \geq f^{+} \circ i d_{M} \circ f \text { since } g \in \operatorname{Res}(M, P) \\
& =f^{+} \circ f \text { since } i d_{M} \text { is the identity map of } M \\
& \geq i d_{L} \text { since } f \in \operatorname{Res}(L, M) .
\end{aligned}
$$




$$
\begin{aligned}
(g \circ f) \circ\left(f^{+} \circ g^{+}\right) & =g \circ\left(f \circ f^{+}\right) \circ g^{+} \\
& \leq g \circ i d_{M} \circ g^{+} \text {since } f \in \operatorname{Res}(L, M) \\
& =g \circ g^{+} \text {since } i d_{M} \text { is the idenitity map of } M \\
& \leq i d_{P} \text { since } g \in \operatorname{Res}(M, P) .
\end{aligned}
$$

Thus, $g \circ f$ is isotone and there exists isotone map $(g \circ f)^{+}=f^{+} \circ g^{+}$that satisfies the definition, so $g \circ f \in \operatorname{Res}(L, P)$.

We want to consider the residuated maps from a lattice $L$ to a lattice $M$, which we will denote $\operatorname{Res}(L, M)$. In particular, we will discuss the residuated maps from a lattice $L$ to itself, denoted $\operatorname{Res}(L)$, and we will restrict $L$ to be finite and nondistributive as this case has not been studied before. The technical definition above does not make it clear how we can construct residuated maps on a finite (and thus complete) lattice. To do so more elegantly, we consider the following lemma.

LEMMA 2.3. A mapping $f: L \rightarrow M$ where $L$ and $M$ are complete lattices is a residuated map if and only if $f$ is a complete join-homomorphism. Moreover, $f\left(0_{L}\right)=0_{M}$

Proof. $(\Rightarrow)$ Assume $f: L \rightarrow M$ is a residuated map where $L$ and $M$ are complete lattices. Let $A$ be a nonempty subset of $L$. Let $y=\bigvee\{f(a) \mid a \in A\}=\bigvee f(A)$, which we know exists since $L$ and $M$ are complete lattices. Then $y \geq f(a)$ for all $a \in A$ and thus $f^{+}(y) \geq f^{+}(f(a)) \geq a$ for all $a \in A$. This implies $f^{+}(y)$ is an upper bound of $A$, and thus $f^{+}(y) \geq \bigvee A$. But then we have $y \geq f\left(f^{+}(y)\right) \geq f(\bigvee A)$ and thus $\bigvee f(A) \geq f(\bigvee A)$.

We get the reverse inequality for free; since $a \leq \bigvee A$ for all $a \in A$, we have $f(a) \leq f(\bigvee A)$ but then $\bigvee f(A) \leq f(\bigvee A)$. Thus, $\bigvee f(A)=f(\bigvee A)$ and $\mathrm{f}$ is a complete join-homomorphism.

$(\Leftarrow)$ Assume now that $f: L \rightarrow M$ is a complete join-homomorphism. Note 
that $f$ is isotone, since if $x \leq y \in L$, then $f(x \vee y)=f(y)=f(x) \vee f(y) \Longrightarrow f(x) \leq$ $f(y)$. To see $f$ is residuated, define its residual $f^{+}: M \rightarrow L$ by $f^{+}(y)=\bigvee\{x \in L$ $f(x) \leq y\}$. Then for $a \in L$, we see $f^{+}(f(a))=\bigvee\{x \in L \mid f(x) \leq f(a)\} \geq a$. And for $b \in M$, we see $f\left(f^{+}(b)\right)=f(\bigvee\{x \in L \mid f(x) \leq b\})=\bigvee f(\{x \in L \mid f(x) \leq b\} \leq$ b. So $f$ is residuated with $f^{+}$its residual.

It is easy to see that $f\left(0_{L}\right)=0_{M}$ since $0_{L} \leq f^{+}\left(0_{M}\right)$ and thus $f\left(0_{L}\right) \leq$ $f\left(f^{+}\left(0_{M}\right)\right)=0_{M}$

It is not difficult to show that $\operatorname{Res}(L, M)$ and $\operatorname{Res}(L)$ are lattices as well, with bottom element the all-0 map, with top element the map that sends every nonzero element to 1 , with the join being a pointwise join, and with the meet being the join of all the lower bounds.

Of particular importance are some step functions in $\operatorname{Res}(L, M)$ which will become vital in our discussion of order automorphisms later. We will call these residuated maps the "deltas" of $\operatorname{Res}(L, M)$, and note that they are equivalent to Schreiner's basic tight maps [23].

DEFINITION 2.14 ( $\delta_{a, b}$ maps). Let $L$ and $M$ be lattices. For any $a \in L$ and $b \in M$,

$$
\delta_{a, b}(x)=\left\{\begin{array}{rr}
0 & \text { if } x \leq a \\
b & \text { otherwise. }
\end{array}\right.
$$

is a residuated map. It's residual, which we denote $\delta_{a, b}^{+}$, is defined as

$$
\delta_{a, b}^{+}(x)=\left\{\begin{array}{rr}
1 & \text { if } b \leq x \\
a & \text { otherwise. }
\end{array}\right.
$$

Note, for clarity, that $\delta_{a, b} \in \operatorname{Res}(L, M)$ but $\delta_{a, b}^{+}$is its residual, and in general is not a residuated map. These delta maps are identical in concept to the basic tight maps Schreiner describes in [23] as well as the $\delta$ maps discussed in [21] and therefore are anticipated here. In the distributive case, every join-irreducible is a $\delta$ whereas there are some non- $\delta$ join-irreducibles in the nondistributive case. 
LEMMA 2.4. Let $L, M$ be finite lattices and $\operatorname{Res}(L, M)$ be the lattice of residuated maps from $L$ to $M$. Then $\delta_{a, b} \in \operatorname{Res}(L, M)$ is join-irreducible if and only if a is meet-irreducible in $L$ and $b$ is join-irreducible in $M$.

Proof. $(\Rightarrow)$ Suppose $\delta_{a, b}$ is join-irreducible in $\operatorname{Res}(L, M)$. For sake of contradiction, suppose there exist $a_{1}, a_{2} \in L$ such that $a=a_{1} \wedge a_{2}$ with $a \neq a_{1}$ and $a \neq a_{2}$. Then $\delta_{a, b}=\delta_{a_{1}, b} \vee \delta_{a_{2}, b}$, contradicting the fact that $\delta_{a, b}$ is join-irreducible. Thus, $a$ is meet-irreducible in $L$. Similarly, suppose for sake of contradiction that there exist $b_{1}, b_{2} \in M$ such that $b=b_{1} \vee b_{2}$ with $b \neq b_{1}$ and $b \neq b_{2}$. Then $\delta_{a, b}=\delta_{a, b_{1}} \vee \delta_{a, b_{2}}$, contradicting the fact that $\delta_{a, b}$ is join-irreducible. Thus, $b$ is join-irreducible in $M$.

$(\Leftarrow)$ Suppose $a$ is meet-irreducible in $L$ and $b$ is join-irreducible in $M$. For sake of contradiction, suppose $\delta_{a, b}=f \vee g$ where $f, g \in \operatorname{Res}(L, M)$ and $f \neq \delta_{a, b} \neq g$. Then since $b$ is join-irreducible and $f \neq g$, there exist $u, v \in L$ so that

$$
\begin{array}{ll}
f(u)<b & g(u)=b \\
f(v)=b & g(v)<b
\end{array}
$$

Furthermore, note that $u$ and $v$ are non-comparable and neither $u$ nor $v$ are less than $a$ since $f$ and $g$ are non-decreasing. Now we have

$$
\delta_{a, b}(u \wedge v)=(f \vee g)(u \wedge v)=f(u \wedge v) \vee g(u \wedge v) \leq f(u) \vee g(v)<b .
$$

So, since $\delta_{a, b}$ takes on only the values 0 and $b$, this implies that $\delta_{a, b}(u \wedge v)=$ $0=f(u)=g(v)$ and, furthermore, that $u \wedge v \leq a$. Since $f(a)=0=f(u)$ and $g(a)=0=g(v)$, we have that $f(a \vee u)=0=g(a \vee v)$. Because $a$ is meet-irreducible and is a lower bound of both $a \vee u$ and $a \vee v$, we have then that $(a \vee u) \wedge(a \vee v)>a$. Thus, $\delta_{a, b}$ 's value on this element must be $b$.

$$
\delta_{a, b}((a \vee u) \wedge(a \vee v))=f((a \vee u) \wedge(a \vee v)) \vee g((a \vee u) \wedge(a \vee v))=b
$$


However,

$$
\begin{aligned}
& f((a \vee u) \wedge(a \vee v)) \leq f(a \vee u)=0 \\
& g((a \vee u) \wedge(a \vee v)) \leq g(a \vee v)=0
\end{aligned}
$$

contradicting the fact that $\delta_{a, b}=f \vee g$.

Since the collection of residuated maps from $L$ to $M$ is a lattice itself, we can discuss the lattice order automorphisms of $\operatorname{Res}(L, M)$. A quick observation is that for every $f \in \operatorname{Res}(L, M)$, the map $\Gamma_{1}=\theta \circ f \circ \gamma$ where $\theta$ is an order automorphism of $M$ and $\gamma$ is an order automorphism of $L$ is an order automorphism of $\operatorname{Res}(L, M)$. Similarly, if $L$ and $M$ are dual lattices, then $\Gamma_{2}=\alpha \circ f^{+} \circ \beta$ where $\alpha$ and $\beta$ are order isomorphisms from $L$ to $M^{\partial}$, the dual of $M$, is an order automorphism of $\operatorname{Res}(L, M)$. These two types of maps are what we will call "induced" order automorphisms, as they are induced by left-right composition. The below lemma proves the two types create distinct order automorphisms, given $L$ and $M$ are duals of each other.

LEMMA 2.5. Let $L$ and $M$ be dual lattices and not both the 2-chain. Let Res $(L, M)$ be the lattice of residuated maps from $L$ to $M$. Let $\theta \in \operatorname{Aut}(M)$ and $\gamma \in \operatorname{Aut}(L)$ and $\alpha, \beta$ be order isomorphisms from $L$ to $M^{\partial}$. Then there exists $f \in \operatorname{Res}(L, M)$ with residual $f^{+}$such that $\theta \circ f \circ \gamma \neq \alpha \circ f^{+} \circ \beta$. In other words, left-right induced order automorphisms of $\operatorname{Res}(L, M)$ using $f \in \operatorname{Res}(L, M)$ and left-right induced order automorphisms of Res $(L, M)$ using its residual $f^{+}$are distinct.

Proof. Denote $\Gamma_{1}\left(\delta_{a, b}\right)=\left(\theta \circ \delta_{a, b} \circ \gamma\right)(x)$ and $\Gamma_{2}\left(\delta_{a, b}\right)=\left(\alpha \circ \delta_{a, b}^{+} \circ \beta\right)(x)$. Now consider the actions of $\Gamma_{1}$ and $\Gamma_{2}$ on $\delta_{a, b}$ where $a \in L$ and $b \in M$ :

$$
\Gamma_{1}\left(\delta_{a, b}\right)(x)=\left(\theta \circ \delta_{a, b} \circ \gamma\right)(x)=\left\{\begin{array}{lr}
0 & \text { if } x \leq \gamma^{-1}(a) \\
\theta(b) & \text { otherwise }
\end{array}\right\}=\delta_{\gamma^{-1}(a), \theta(b)}(x)
$$

and, similarly, $\Gamma_{2}\left(\delta_{a, b}\right)(x)=\delta_{\beta^{-1}(b), \alpha(a)}(x)$. Without loss of generality, let $b$ be neither the top or bottom element of $M$ (if $M$ is the 2-chain, instead consider the 
case where $a$ is neither the top or bottom element of $L$ and look at $\left.\delta_{a, 1}\right)$. We have $\Gamma_{1}\left(\delta_{0, b}\right)=\delta_{0, \theta(b)}$ whereas $\Gamma_{2}\left(\delta_{0, b}\right)=\delta_{\beta^{-1}(b), 1}$, which are not equal. Thus, $\Gamma_{1} \neq \Gamma_{2}$.

This result is important because our argument will depend on counting the number of induced order automorphisms of $\operatorname{Res}\left(M_{3}\right)$ and $\operatorname{Res}\left(N_{5}\right)$, and since $M_{3}$ and $N_{5}$ are both self-dual, Lemma 2.5 ensures we do not double count any order automorphism.

The definitions and lemmas of this chapter form a solid base from which the remaining chapters are built. Next, we will discuss the residuated maps on the lattice $M_{n}$ and show that the order automorphisms of the lattice of these maps are indeed all induced by left-right composition. 


\section{CHAPTER 3 \\ ORDER AUTOMORPHISMS OF RES $\left(M_{n}\right)$}

To reiterate our goal: we want to begin with a finite nondistributive lattice $L$ and look at the residuated maps from $L$ to itself, denoted $\operatorname{Res}(L)$. The set of maps $\operatorname{Res}(L)$ form their own lattice using pointwise partial order, and so we will study the order automorphisms of this lattice, which we denote $\operatorname{Aut}(\operatorname{Res}(L))$. We specifically want to investigate if these order automorphisms are all of the induced type discussed in Chapter 2. Because nondistributive lattices are characterized by having one or both of $M_{3}$ and $N_{5}$ as sublattices, we begin our investigation by exploring the residuated maps of $M_{n}$, for which $M_{3}$ is a special case.

\subsection{Elements of $\operatorname{Res}\left(M_{n}\right)$}

Let us first discuss the number and types of residuated maps on the lattice $M_{n}$. For convenience, we will denote the atoms of $M_{n}$ as $\left\{a_{1}, a_{2}, \ldots, a_{n}\right\}$ (see Fig. 2.1). If $f$ is a residuated map on $M_{n}$, then $f$ is determined by its values on the atoms since $f(0)=0$ and $f(1)=f\left(a_{i}\right) \vee f\left(a_{j}\right)$ for $i \neq j$. We will use "slot notation" to describe functions in $\operatorname{Res}\left(M_{n}\right)$, using ordered slots for the image of each atom; i.e. $\left(f\left(a_{1}\right) f\left(a_{2}\right) \ldots f\left(a_{n}\right)\right)$ will describe $f \in \operatorname{Res}\left(M_{n}\right)$. For instance, if $f \in \operatorname{Res}\left(M_{2}\right)$ with $f(0)=0, f\left(a_{1}\right)=a_{2}, f\left(a_{2}\right)=a_{1}$, and $f(1)=1$, we will denote $f$ in slot notation as $\left(a_{2} a_{1}\right)$.

THEOREM 3.1. The number of residuated maps from $M_{n}$ to $M_{n}$ is given by the 
formula

$$
1+(n+1)^{2}+n !+\sum_{k=1}^{n-1}\left(\begin{array}{l}
n \\
k
\end{array}\right){ }_{n} P_{n-k} .
$$

Proof. We will describe and count the different types of functions in $\operatorname{Res}\left(M_{n}\right)$.

Type 1: [ $\delta$ functions $]$ The $\delta$ functions defined in Definition 2.14 can be characterized in three subtypes.

(i) The "constant" $\delta$ maps: Though we call these maps "constant," we really mean the image of every nonzero element is the same. The constant maps include the all-zero map $\left(\delta_{x, 0}\right.$ or $\left.\delta_{1, y}\right)$, the map that sends every nonzero element to $1\left(\delta_{0,1}\right)$, and the maps $\delta_{0, a_{i}}$ which send every nonzero element to the atom $a_{i}$. We write these maps in $\operatorname{Res}\left(M_{3}\right)$ slot notation as (000), (111), and $\left(a_{i} a_{i} a_{i}\right)$. There are a total of $n+2$ of these "constant" $\delta$ maps in $\operatorname{Res}\left(M_{n}\right)$.

(ii) The $\delta$ join-irreducibles: The maps $\delta_{a_{i}, a_{j}}$ send one atom to 0 and send the remaining atoms to a single atom. For example, the map $\delta_{a_{1}, a_{3}}$ in $\operatorname{Res}\left(M_{3}\right)$ is written as $\left(0 a_{3} a_{3}\right)$ in slot notation and it sends $a_{1}$ to 0 while sending $a_{2}$ and $a_{3}$ both to $a_{3}$. By Lemma 2.4, the $\delta_{a_{i}, a_{j}}$ maps are join-irreducibles of $\operatorname{Res}\left(M_{n}\right)$ since $\left\{a_{1}, a_{2}, \ldots, a_{n}\right\}$ are both join-irreducible and meet-irreducible elements of $M_{n}$. There are $n^{2}$ of these maps in $\operatorname{Res}\left(M_{n}\right)$.

(iii) The 0,1- $\delta$ maps: The maps $\delta_{a_{i}, 1}$ have only 0 and 1 as images. These maps are the join of two $\delta$ join-irreducibles. For instance, the map $(011)=$ $\delta_{a_{1}, 1}=\delta_{a_{1}, a_{2}} \vee \delta_{a_{1}, a_{3}}$ in $\operatorname{Res}\left(M_{3}\right)$. There are $n$ of these maps in $\operatorname{Res}\left(M_{n}\right)$.

We can count that there are a total of $n^{2}+2 n+2=1+(n+1)^{2}$ Type 1 functions in $\operatorname{Res}\left(M_{n}\right)$.

Type 2: $\left[a_{i}, 1\right.$ functions] Functions that map up to $n-1$ of the atoms to 1 and the remaining to distinct atoms are easily shown to be in $\operatorname{Res}\left(M_{n}\right)$. For instance, the map $f=\left(1 a_{1} a_{2}\right) \in \operatorname{Res}\left(M_{3}\right)$ because $f(1)=f\left(a_{1}\right) \vee f\left(a_{2}\right)=$ 
$f\left(a_{1}\right) \vee f\left(a_{3}\right)=f\left(a_{2}\right) \vee f\left(a_{3}\right)$, so the map is join-preserving. It is necessary for the elements to be distinct, or the map is not residuated. For instance, the map $\left(1 a_{1} a_{1}\right) \notin \operatorname{Res}\left(M_{3}\right)$ because $f(1)=f\left(a_{1}\right) \vee f\left(a_{2}\right)=1$ contradicts $f(1)=f\left(a_{2}\right) \vee f\left(a_{3}\right)=a_{1}$.

We map only $n-1$ of the atoms to 1 to avoid double counting $\delta_{0,1}$, which we included in the Type 1 maps. To count the Type 2 maps, we need to consider separate cases based on how many of the atoms are mapped to 1 , so we have to count a sum from 1 to $n-1$ of $k$ atoms mapped to 1 . In each case, we then have $n$ choose $k$ ways to place the 1 s and then we pick $n-k$ elements from $n$ distinct atoms as the image of the others. This gives us a count of $\sum_{k=1}^{n-1}\left(\begin{array}{l}n \\ k\end{array}\right)_{n} P_{n-k}$ Type 2 maps in $\operatorname{Res}\left(M_{n}\right)$.

Type 3: [permutations] These functions map the atoms to permutations of themselves. The permutations are easily seen to be residuated maps, since permutations are lattice homomorphisms and thus join-homomorphisms. The permutations of $\operatorname{Res}\left(M_{3}\right)$ are written in slot notation as $\left(a_{1} a_{2} a_{3}\right),\left(a_{1} a_{3} a_{2}\right)$, $\left(a_{2} a_{1} a_{3}\right),\left(a_{2} a_{3} a_{1}\right),\left(a_{3} a_{1} a_{2}\right)$, and $\left(a_{3} a_{2} a_{1}\right)$. Note again that all of the images must be distinct atoms in this Type 3 case. Since there are $n$ ! permutations of $n$ objects, we have that there are $n$ ! Type 3 functions in $\operatorname{Res}\left(M_{n}\right)$.

Finally, note that Types 1 through 3 are all mutually exclusive and take into account every possible map of the atoms that preserves joins, and thus every residuated map. By adding together our counts of each type of mapping, we arrive at the result:

$$
\left|\operatorname{Res}\left(M_{n}\right)\right|=1+(n+1)^{2}+n !+\sum_{k=1}^{n-1}\left(\begin{array}{l}
n \\
k
\end{array}\right){ }_{n} P_{n-k} .
$$

Table 3.1 includes some calculations of the size of $\operatorname{Res}\left(M_{n}\right)$ for small values of $n$. We have searched the Online Encyclopedia of Integer Sequences [12], and it seems the count of residuated maps on $M_{n}$ is a new integer sequence. 
Table 3.1: Size of $\operatorname{Res}\left(M_{n}\right)$

\begin{tabular}{|c|c|}
\hline$n$ & $\left|\operatorname{Res}\left(M_{n}\right)\right|$ \\
\hline 3 & 50 \\
\hline 4 & 424 \\
\hline 5 & 1,582 \\
\hline 7 & 130,986 \\
\hline 10 & $234,662,352$ \\
\hline 20 & $\approx 1.7 \times 10^{21}$ \\
\hline
\end{tabular}

For completeness sake and as an exercise in building the lattice of residuated maps discussed in this section, the reader can refer to Figure 3.1 and to Figure 3.2 for Hasse diagrams of $\operatorname{Res}\left(M_{2}\right)$ and $\operatorname{Res}\left(M_{3}\right)$, which were constructed using Maple.

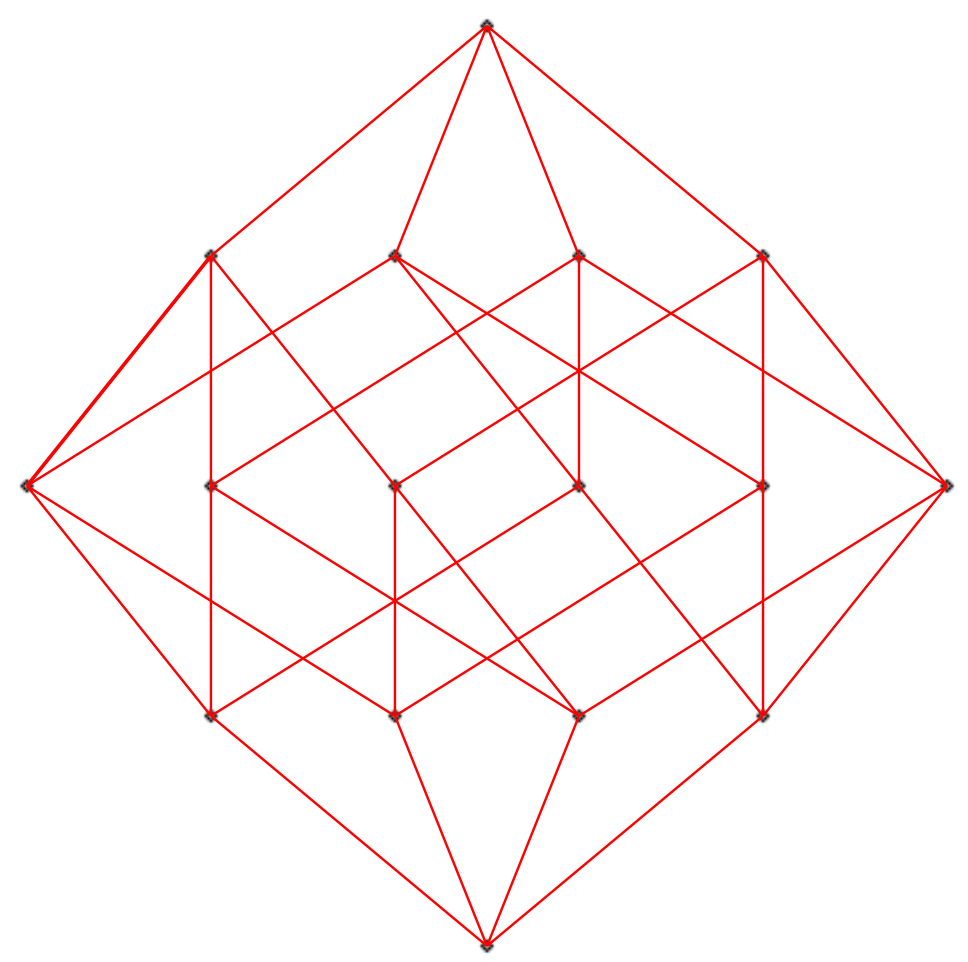

Figure 3.1: $\operatorname{Res}\left(M_{2}\right)$ 


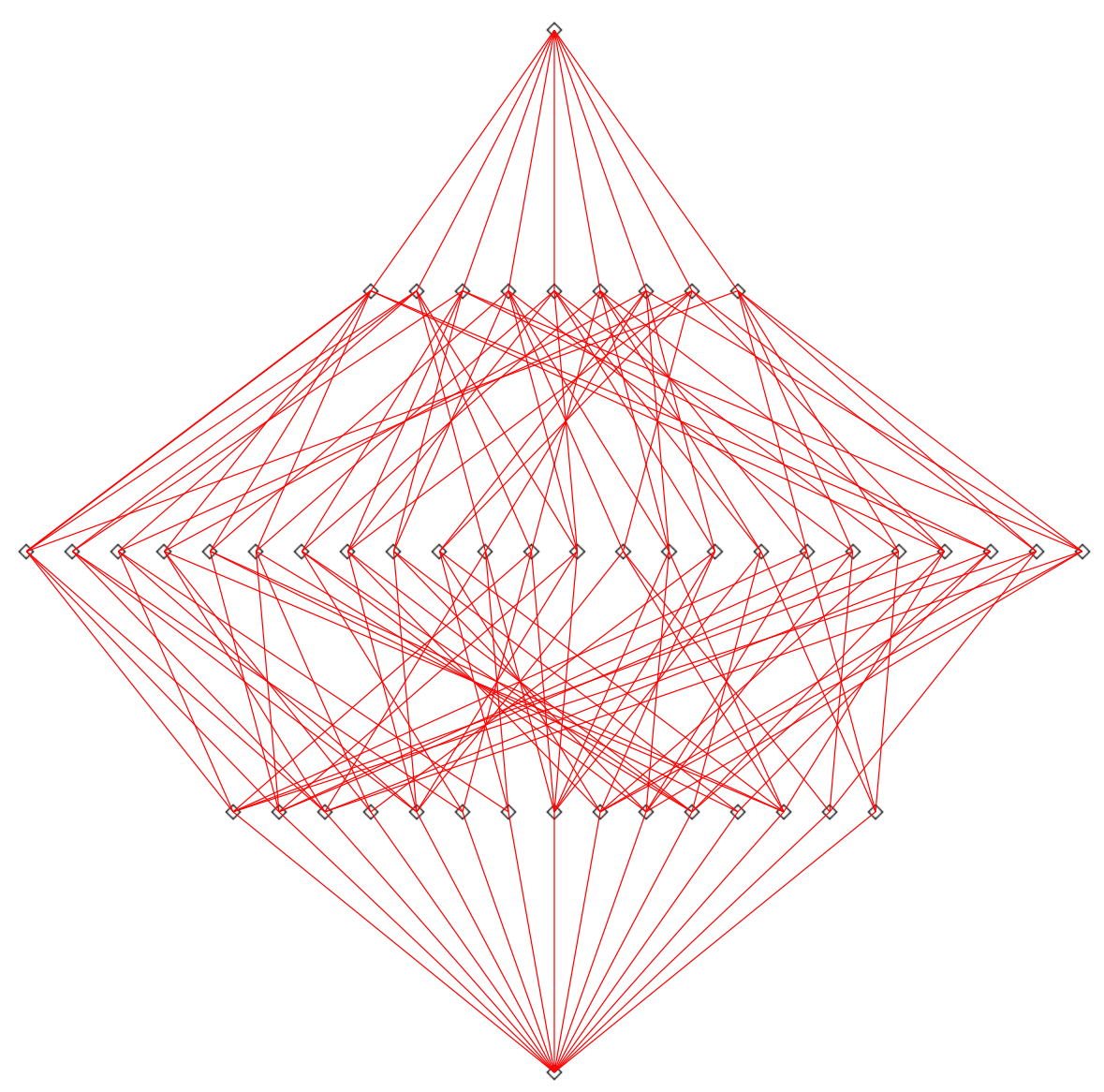

Figure 3.2: $\operatorname{Res}\left(M_{3}\right)$

\subsection{Join-Irreducibles of $\operatorname{Res}\left(M_{n}\right)$}

In the proof above, we observed briefly the three types of elements in $\operatorname{Res}\left(M_{n}\right)$. We now examine these types one more time in order to identify the join-irreducibles of $\operatorname{Res}\left(M_{n}\right)$. Please note that join-irreducibles for $\operatorname{Res}\left(M_{2}\right)$ and $\operatorname{Res}\left(M_{3}\right)$ are slightly different than those of $\operatorname{Res}\left(M_{n}\right)$ for $n>3$. The majority of the reasoning below ties back to the atoms $\left\{a_{1}, a_{2}, \ldots, a_{n}\right\}$ being join-irreducibles of $M_{n}$.

Type 1: [ $\delta$ functions $]$ As previously discussed, by Lemma 2.4, the only $\delta$ functions that are join-irreducible are of the form $\delta_{a_{i}, a_{j}}$.

Type 2: $\left[a_{i}, 1\right.$ functions $]$ The Type 2 functions are not join-irreducible for 
$\operatorname{Res}\left(M_{2}\right)$ and $\operatorname{Res}\left(M_{3}\right)$ because they are either the join of two $\delta$ functions, or the join of two other Type 2 functions. For instance, in $\operatorname{Res}\left(M_{3}\right)$ we have $\left(a_{1} a_{2} 1\right)=\left(0 a_{2} a_{2}\right) \vee\left(a_{1} 0 a_{1}\right)=\delta_{a_{1}, a_{2}} \vee \delta_{a_{2}, a_{1}}$ while $\left(a_{1} 11\right)=\left(a_{1} a_{2} 1\right) \vee\left(a_{1} a_{3} 1\right)$

However, for $n>3$, the Type 2 functions do include some join-irreducibles. In the $n>3$ case, maps with one atom mapped to 1 , such as $\left(1 a_{2} a_{3} \ldots a_{n}\right)$, are join-irreducible because the only nonzero element below them is a single permutation, in this instance $\left(a_{1} a_{2} a_{3} \ldots a_{n}\right)$. We will call these the single-1 join-irreducibles.

Type 3: [permutations] By [23] and [25], since $M_{2}$ is distributive and thus $\operatorname{Res}\left(M_{2}\right)$ is distributive, we have that the only join-irreducibles of $\operatorname{Res}\left(M_{2}\right)$ are the $\delta$ join-irreducibles. However, $M_{n}$ with $n \geq 3$ is nondistributive, and hence $\operatorname{Res}\left(M_{n}\right)$ is nondistributive and must have join-irreducible elements besides the $\delta$ join-irreducibles. Some of these are the permutations, since the only element below a permutation in $\operatorname{Res}\left(M_{n}\right)$ for $n \geq 3$ is the all-zero map.

We summarize the join-irreducibles of $\operatorname{Res}\left(M_{n}\right)$ in Table 3.2.

Table 3.2: Join-Irreducibles of $\operatorname{Res}\left(M_{n}\right)$

\begin{tabular}{|c|c|c|}
\hline Lattice & Join-Irreducibles & Example \\
\hline $\operatorname{Res}\left(M_{2}\right)$ & $\delta$ join-irreducibles & $\left(0 a_{1}\right)$ \\
\hline $\operatorname{Res}\left(M_{3}\right)$ & $\delta$ join-irreducibles & $\left(0 a_{1} a_{1}\right)$ \\
& permutations & $\left(a_{2} a_{3} a_{1}\right)$ \\
\hline $\operatorname{Res}\left(M_{n}\right), n>3$ & $\delta$ join-irreducibles & $\left(0 a_{1} a_{1} \ldots a_{1}\right)$ \\
& $\begin{array}{c}\text { permutations } \\
\left(a_{2} a_{3} \ldots a_{n} a_{1}\right) \\
\end{array}$ & $\left(1 a_{2} a_{3} \ldots a_{n}\right)$ \\
\hline
\end{tabular}


Note: The change in structure between $\operatorname{Res}\left(M_{3}\right)$ and $\operatorname{Res}\left(M_{4}\right)$ produces an interesting result. In $\operatorname{Res}\left(M_{n}\right)$ for $n>3$, the single-1 join-irreducibles are the only cover of a corresponding permutation join-irreducible. This creates several sublattices isomorphic to $N_{5}$ in $\operatorname{Res}\left(M_{n}\right)$ for $n>3$. Thus, we have an example of a lattice $L$ for which $L$ is modular but $\operatorname{Res}(L)$ is nonmodular. This is particularly surprising, because [25] has proved that $L$ is distributive if and only if $\operatorname{Res}(L)$ is distributive. See Figures 3.3 and 3.4 for a representation of these different structures. You will see in Figure 3.4 a copy of $N_{5}$ highlighted in blue.

LEMMA 3.1. If $L$ is a finite modular lattice, it is not necessarily the case that $\operatorname{Res}(L)$ is modular.

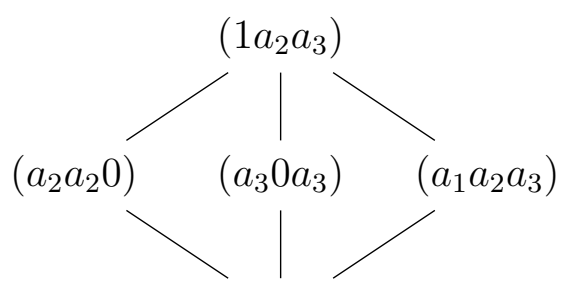

(000)

Figure 3.3: Partial Structure of Res $\left(M_{3}\right)$

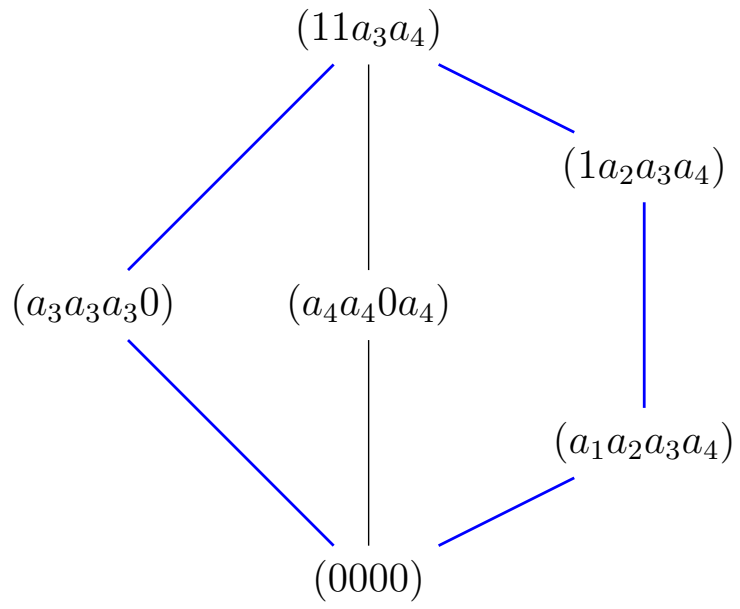

Figure 3.4: Partial Structure of Res $\left(M_{4}\right)$ 


\subsection{Order Automorphisms of Res $\left(M_{2}\right)$}

In discussing order automorphisms of $\operatorname{Res}\left(M_{n}\right)$, we will begin by discussing those of $\operatorname{Res}\left(M_{2}\right)$. This case is different than the remaining cases since $M_{2}$ is distributive while $M_{n}$ for $n \geq 3$ is not. In this regard, it is not surprising that this case yields different results. We can quickly show that the order automorphisms of $\operatorname{Res}\left(M_{2}\right)$ are not all induced.

First, note that since $M_{2}$ is distributive, $\operatorname{Res}\left(M_{2}\right)$ is also distributive [25]. Also, as a finite distributive lattice, every element of $\operatorname{Res}\left(M_{2}\right)$ can be uniquely written (up to order) as the join of the maximal join-irreducibles below it. So to determine the order automorphisms of $\operatorname{Res}\left(M_{2}\right)$, one needs only to consider where such functions can map the join-irreducibles. From our discussion in Section 3.1, we can determine that there are only four join-irreducibles of $\operatorname{Res}\left(M_{2}\right)$ (they are the set $\left.\left\{\left(a_{1} 0\right),\left(a_{2} 0\right),\left(0 a_{1}\right),\left(0 a_{2}\right)\right\}\right)$, and this set of join-irreducibles forms an antichain. Using Birkhoff's Representation Theorem [5], we know also that $\operatorname{Res}\left(M_{2}\right) \cong 2^{J\left(\operatorname{Res}\left(M_{2}\right)\right)}$, the non-decreasing functions from $J\left(\operatorname{Res}\left(M_{2}\right)\right)$ into the 2chain. In this case, it turns out this is isomorphic to the power set on four elements since the join-irreducibles form a four-element antichain. Thus, there are $4 !=24$ order automorphisms of $\operatorname{Res}\left(M_{2}\right)$.

On the other hand, there are only 8 induced order automorphisms of $\operatorname{Res}\left(M_{2}\right)$. There are two automorphisms of $M_{2}$, and thus a total of four ways to choose $\theta$ and $\gamma$ to induce the order automorphisms $\theta \circ f \circ \gamma$ with $f \in \operatorname{Res}\left(M_{2}\right)$. There are also two dual isomorphisms of $M_{2}$, and thus a total of four ways to choose $\alpha$ and $\beta$ to induce the order automorphisms $\alpha \circ f^{+} \circ \beta$ with $f^{+}$the dual of $f \in \operatorname{Res}\left(M_{2}\right)$. That makes for 8 total induced order automorphisms of $\operatorname{Res}\left(M_{2}\right)$. Hence, since there are 24 order automorphisms of $\operatorname{Res}\left(M_{2}\right)$ but only 8 are induced, we conclude not all order automorphisms are induced.

One can observe how the induced automorphisms act on the join-irreducibles 
of $M_{2}$, since there are only 8 ways to do so. Then an example of an order automorphism that is not induced becomes readily available: the order automorphism that sends $\delta_{b, a} \rightarrow \delta_{a, a}$ and $\delta_{a, a} \rightarrow \delta_{b, a}$ but leaves $\delta_{a, b}$ and $\delta_{b, b}$ fixed is not induced.

\subsection{Order Automorphisms of $\operatorname{Res}\left(M_{n}\right)$ for $n \geq 3$}

The cases for $\operatorname{Res}\left(M_{n}\right)$ where $n \geq 3$, the nondistributive lattices, are more complicated and also somewhat surprising. We can see, through some investigation of the elements described in Table 3.2, that the join-irreducibles of $\operatorname{Res}\left(M_{n}\right)$ are not connected as a partially ordered set. One might expect then that the order automorphisms are not induced, similar to the results on $\operatorname{Res}\left(M_{2}\right)$ where the joinirreducibles form an antichain. However, the order automorphisms for $\operatorname{Res}\left(M_{n}\right)$ where $n \geq 3$ are indeed all induced, so it seems distributivity may play a key role in the automorphisms of residuated maps. We will prove some general results first that will help to prove one of our main theorems, alongside which we will also discuss the slight differences between the $\operatorname{Res}\left(M_{3}\right)$ case and the $\operatorname{Res}\left(M_{n}\right), n>3$ case.

For both the $\operatorname{Res}\left(M_{3}\right)$ and more general $\operatorname{Res}\left(M_{n}\right), n>3$ case, the key observation is that determining the $\delta$ join-irreducibles determines the entire order automorphism. The first step in proving this is noting that $\delta$ join-irreducibles must map to $\delta$ join-irreducibles, which we prove in the following lemma.

LEMMA 3.2. For $n \geq 3$, if $\Gamma \in \operatorname{Aut}\left(\operatorname{Res}\left(M_{n}\right)\right)$ and $\delta_{a_{i}, a_{j}} \in \operatorname{Res}\left(M_{n}\right)$, then $\Gamma\left(\delta_{a_{i}, a_{j}}\right)=\delta_{a_{k}, a_{l}}$. Furthermore, if $\Gamma(f)=\delta_{a_{i}, a_{j}}$, then $f$ is a $\delta$ join-irreducible. That is, an order automorphism of Res $\left(M_{n}\right)$ keeps the set of $\delta$ join-irreducibles invariant.

Proof. By careful examination of the elements discussed in Section 3.1, we can determine that the atoms of $\operatorname{Res}\left(M_{n}\right)$ for $n \geq 3$ are the $\delta$ join-irreducibles and the permutation join-irreducibles. An order automorphisms has to preserve covering relations 
and thus must map atoms to atoms, so we know $\delta$ join-irreducibles of $\operatorname{Res}\left(M_{n}\right)$ can only map to either a $\delta$ join-irreducible or a permutation join-irreducible. We can further limit the possibilities by noting that the $\delta$ join-irreducibles and permutation join-irreducibles have a different number of covers.

We again refer to the types of functions discussed in Section 3.1 to investigate the covers of these elements. For generality's sake, let us count the covers of $\delta_{a_{i}, a_{j}}$ in $\operatorname{Res}\left(M_{n}\right)$ for $n \geq 3$. There are two Type $1 \delta$ functions that cover this element: $\delta_{0, a_{j}}$ and $\delta_{a_{i}, 1}$. We count the Type 2 functions that cover $\delta_{a_{i}, a_{j}}$ by noting that these covers will map $a_{i}$ to any atom besides $a_{j}$ and then map all but one of the remaining atoms to 1 , leaving the last atom to map to $a_{j}$. (For example, $\left(a_{1} a_{2} 11\right)$ covers $\left(0 a_{2} a_{2} a_{2}\right)$ in $\operatorname{Res}\left(M_{4}\right)$.) This gives us $(n-1)(n-1)$ Type 2 functions that cover $\delta_{a_{i}, a_{j}}$. There are no Type 3 permutation functions that cover $\delta_{a_{i}, a_{j}}$. Thus, there are a total of $(n-1)(n-1)+2=n^{2}-2 n+3$ covers of $\delta_{a_{i}, a_{j}}$.

On the other hand, let us count the covers of a permutation join-irreducible. There are no Type $1 \delta$ functions that cover any permutation since permutations take on all $a_{i}$ value whereas $\delta$ functions can only take on a single $a_{i}$ value. To count the Type 2 functions that cover a permutation, note that these covers will be equal to the permutation at all but one atom, and will map this atom instead to 1 . That gives a count of $n$ Type 2 functions that cover a given permutation. There are no Type 3 functions that cover a permutation, since these functions are permutations themselves and the permutations form an antichain. Thus, there are a total of $n$ covers of a permutation join-irreducible in $\operatorname{Res}\left(M_{n}\right)$ for $n \geq 3$.

There is no integer that satisfies $n^{2}-2 n+3=n$, and so we conclude that the $\delta$ join-irreducibles and the permutation join-irreducibles are covered by a different number of elements in $\operatorname{Res}\left(M_{n}\right)$ for $n \geq 3$. Note also that the additional single1 join-irreducibles found in $\operatorname{Res}\left(M_{n}\right)$ for $n \geq 4$ are not atoms, and so are not of concern to us here. Thus, since order automorphisms preserve covering relations, we 
can conclude that $\delta$ join-irreducibles map to $\delta$ join-irreducibles and, furthermore, that permutation join-irreducibles map to permutation join-irreducibles.

The proof that every preimage of a $\delta_{a_{i}, a_{j}}$ is also a $\delta$ join-irreducible is then given to us without any extra work, as $\Gamma$ is a bijection and the set of $\delta$ joinirreducibles is finite.

Now, we will count the ways that an order automorphism of $\operatorname{Res}\left(M_{n}\right)$ can map $\delta$ join-irreducibles to one another, which happens to coincide with the number of left-right induced maps. Then, we will prove that an order automorphism is determined by the $\delta$ join-irreducibles and our argument that every order automorphism of $\operatorname{Res}\left(M_{n}\right)$ for $n \geq 3$ is induced by a left-right composition map will be complete.

To further discuss the $\delta$-join irreducibles neatly and concisely, we will introduce some helpful notation and language. First, we will say a set has the " $M_{n}$ property" if any two elements chosen from the set join to the same element, just as the atoms of $M_{n}$ join to 1 . Below we define what we will call "upper- $\Delta$ " and "lower- $\Delta$ " sets, given $a_{i}$ an atom of $M_{n}$ :

$$
\begin{aligned}
& \Delta^{a_{i}}=\left\{\delta_{a_{i}, a_{j}} \mid a_{j} \text { is an atom of } M_{n}\right\} \text { is an upper- } \Delta \text { set; } \\
& \Delta_{a_{i}}=\left\{\delta_{a_{j}, a_{i}} \mid a_{j} \text { is an atom of } M_{n}\right\} \text { is a lower- } \Delta \text { set. }
\end{aligned}
$$

There are $n^{2} \delta$ join-irreducibles in $\operatorname{Res}\left(M_{n}\right)$, and we have now separated them into $2 n$ sets of $n$ elements each. Notice that the upper- $\Delta$ sets are pairwise disjoint (as are the lower- $\Delta$ sets) and that $\Delta^{a_{i}} \cap \Delta_{a_{j}}=\delta_{a_{i}, a_{j}}$.

We have defined our $\Delta$ sets in such a way that they are the only sets containing $n \delta$-join irreducibles with the " $M_{n}$ property." We see this by noting first that $\delta_{a_{i}, a_{j}} \vee \delta_{a_{i}, a_{k}}=\delta_{a_{i}, 1}$ and $\delta_{a_{j}, a_{i}} \vee \delta_{a_{k}, a_{i}}=\delta_{0, a_{i}}$ for any $1 \leq j \neq k \leq n$. Furthermore, these are the maximal sets of $\delta$ join-irreducibles of $\operatorname{Res}\left(M_{n}\right)$ with this property, for suppose we have distinct $\alpha, \beta \in\left\{a_{1}, a_{2}, \ldots, a_{n}\right\}$ and distinct $\gamma, \theta \in\left\{a_{1}, a_{2}, \ldots, a_{n}\right\}$, 
then we have the following join:

$$
\left(\delta_{\alpha, \gamma} \vee \delta_{\beta, \theta}\right)(x)= \begin{cases}0 & x \leq \alpha, x \leq \beta \\ \gamma & x \not \leq \alpha, x \leq \beta \\ \theta & x \leq \alpha, x \not \leq \beta \\ \gamma \vee \theta & x \not \leq \alpha, x \not \leq \beta\end{cases}
$$

It is clear that the only $\delta$-join irreducibles below this map are the two that joined to form it, and thus no other $\delta$-join irreducibles can be found to make a set with the $M_{n}$ property. Since order automorphisms preserve joins, any automorphism of $\operatorname{Res}\left(M_{n}\right)$ must keep the collection of $\Delta$ sets invariant. We will show that there are $2 \cdot n !^{2}$ ways to do so.

LEMMA 3.3. Suppose $\Gamma \in \operatorname{Aut}\left(\operatorname{Res}\left(M_{n}\right)\right)$ such that $\Gamma\left(\Delta^{x}\right)=\Delta^{y}$. for some $x, y \in$ $\left\{a_{1}, a_{2}, \ldots, a_{n}\right\}$. Then every upper- $\Delta$ set is mapped to an upper $\Delta$ set and every lower- $\Delta$ set is mapped to a lower- $\Delta$ set.

Proof. Without loss of generality, suppose $\Gamma\left(\Delta^{x}\right)=\Delta^{y}$ such that $\Gamma\left(\delta_{x, b_{k}}\right)=\delta_{y, c_{k}}$ where $\left\{b_{1}, b_{2}, \ldots, b_{n}\right\}=\left\{c_{1}, c_{2}, \ldots, c_{n}\right\}=\left\{a_{1}, a_{2}, \ldots, a_{n}\right\}$. Now consider $\Gamma\left(\Delta_{b_{i}}\right)$ by looking at the image of $\delta_{x, b_{i}}$. We have that $\Gamma\left(\delta_{x, b_{i}}\right)=\delta_{y, c_{i}} \in \Delta^{y} \cap \Delta_{c_{i}}$, and thus, since $\Delta^{y}$ is already an image under $\Gamma$, it must be that $\Gamma\left(\Delta_{b_{i}}\right)=\Delta_{c_{i}}$. This proves that the lower- $\Delta$ sets are mapped to lower- $\Delta$ sets, which leaves the upper- $\Delta$ sets to map to upper- $\Delta$ sets.

LEMMA 3.4. Suppose $\Gamma \in \operatorname{Aut}\left(\operatorname{Res}\left(M_{n}\right)\right)$ such that $\Gamma\left(\Delta^{x}\right)=\Delta_{y}$ for some $x, y \in$ $\left\{a_{1}, a_{2}, \ldots, a_{n}\right\}$, the atoms of $M_{n}$. Then every upper- $\Delta$ set is mapped to a lower- $\Delta$ set and vice versa.

Proof. Without loss of generality, suppose $\Gamma\left(\Delta^{x}\right)=\Delta_{y}$ such that $\Gamma\left(\delta_{x, b_{k}}\right)=\delta_{c_{k}, y}$ where $\left\{b_{1}, b_{2}, \ldots, b_{n}\right\}=\left\{c_{1}, c_{2}, \ldots, c_{n}\right\}=\left\{a_{1}, a_{2}, \ldots, a_{n}\right\}$. Then we can find the 
image of $\Delta_{b_{i}}$ under $\Gamma$ by considering its element $\delta_{x, b_{i}}$. Since $\delta_{x, b_{i}} \in \Delta^{x} \cap \Delta_{b_{i}}$, it must be that $\Gamma\left(\delta_{x, b_{i}}\right) \in \Gamma\left(\Delta^{x}\right) \cap \Gamma\left(\Delta_{b_{i}}\right)$. Since $\Gamma\left(\delta_{x, b_{i}}\right)=\delta_{c_{i}, y} \in \Delta^{c_{i}} \cap \Delta_{y}$ and $\Delta_{y}$ is the image of $\Delta^{x}$ under $\Gamma$, we can conclude $\Gamma\left(\Delta_{b_{i}}\right)=\Delta^{c_{i}}$. Thus, every lower- $\Delta$ set is mapped to an upper- $\Delta$ set. Since $\Gamma$ is a bijection and the finite number of $\Delta$ sets map only to each other, we can also conclude that each upper- $\Delta$ set must be mapped to a lower- $\Delta$ set.

We now count the ways an order automorphism keeps the $\Delta$ sets appropriately invariant in the next lemma.

LEMMA 3.5. There are $2 \cdot n !^{2}$ ways for automorphisms of $\operatorname{Res}\left(M_{n}\right)$ to map $\delta$ join-irreducibles to $\delta$ join-irreducibles.

Proof. We have discussed already that an automorphism of $\operatorname{Res}\left(M_{n}\right)$ must map the $\Delta$ sets to one another. Lemmas 3.3 and 3.4 imply that either all upper- $\Delta$ s map to upper- $\Delta \mathrm{s}$ or all upper- $\Delta \mathrm{s}$ map to lower- $\Delta \mathrm{s}$, so we consider two cases.

Case 1: Consider order automorphisms that map all the upper- $\Delta$ sets to upper- $\Delta$ sets. We will show that specifying $\Gamma \in \operatorname{Aut}\left(\operatorname{Res}\left(M_{n}\right)\right)$ on one of $\Delta^{a_{1}}, \Delta^{a_{2}}$, $\ldots, \Delta^{a_{n}}$ determines $\Gamma$ on all of the $\delta$ join-irreducibles. There are $n$ ! ways to choose to map $\Delta^{a_{1}}, \Delta^{a_{2}}, \ldots, \Delta^{a_{n}}$ onto one another and then $n$ ! ways to specify $\Gamma$ on just one of these sets, making for a total of $n !^{2}$ automorphisms that map upper- $\Delta \mathrm{s}$ to upper- $\Delta \mathrm{s}$.

Without loss of generality, suppose $\Gamma \in \operatorname{Aut}\left(\operatorname{Res}\left(M_{n}\right)\right)$ such that $\Gamma\left(\Delta^{a_{i}}\right)=$ $\Delta^{b_{i}}$ for $1 \leq i \leq n$ where $\left\{b_{1}, b_{2}, \ldots, b_{n}\right\}=\left\{a_{1}, a_{2}, \ldots, a_{n}\right\}$. Suppose we specify $\Gamma$ on $\Delta^{a_{1}}$ such that $\Gamma\left(\delta_{a_{1}, a_{j}}\right)=\delta_{b_{1}, c_{j}}$ for $1 \leq j \leq n$ where $\left\{c_{1}, c_{2}, \ldots, c_{n}\right\}=$ $\left\{a_{1}, a_{2}, \ldots, a_{n}\right\}$. Note that lower- $\Delta$ sets map to other lower- $\Delta$ sets in this case. So we have $\Gamma\left(\delta_{a_{i}, a_{j}}\right)=\delta_{b_{i}, c_{j}}$ implies $\Gamma\left(\Delta_{a_{j}}\right)=\Delta_{c_{j}}$. 
To conclude, notice that $\Gamma\left(\delta_{a_{i}, a_{j}}\right) \in \Gamma\left(\Delta^{a_{i}}\right) \cap \Gamma\left(\Delta_{a_{j}}\right)=\Delta^{b_{i}} \cap \Delta_{c_{j}}=\delta_{b_{i}, c_{j}}$. Therefore, every $\delta$ join-irreducible is determined in one of these $n !^{2}$ ways.

Case 2: Consider order automorphisms that map the upper- $\Delta$ sets to the lower- $\Delta$ sets. We will show, again, that specifying $\Gamma \in \operatorname{Aut}\left(\operatorname{Res}\left(M_{n}\right)\right)$ on one of $\Delta^{a_{1}}, \Delta^{a_{2}}, \ldots, \Delta^{a_{n}}$ specifies $\Gamma$ on every $\delta$ join-irreducible. There are $n$ ! ways to map $\Delta^{a_{1}}, \Delta^{a_{2}}, \ldots, \Delta^{a_{n}}$ to $\left\{\Delta_{a_{1}}, \Delta_{a_{2}}, \ldots, \Delta_{a_{n}}\right\}$, and then $n$ ! ways to specify $\Gamma$ on one of these sets. That makes for a total of $n !^{2}$ order automorphisms of $\operatorname{Res}\left(M_{n}\right)$ that map upper- $\Delta$ sets to lower- $\Delta$ sets.

Suppose $\Gamma \in \operatorname{Aut}\left(\operatorname{Res}\left(M_{n}\right)\right)$ such that $\Gamma\left(\Delta^{a_{i}}\right)=\Delta_{b_{i}}$ where $1 \leq i \leq n$ and $\left\{a_{1}, a_{2}, \ldots, a_{n}\right\}=\left\{b_{1}, b_{2}, \ldots, b_{n}\right\}$. Suppose we specify $\Gamma$ on $\Delta^{a_{1}}$ such that $\Gamma\left(\delta_{a_{1}, a_{j}}\right)=\delta_{c_{j}, b_{1}}$ where $1 \leq j \leq n$ and $\left\{a_{1}, a_{2}, \ldots, a_{n}\right\}=\left\{c_{1}, c_{2}, \ldots, c_{n}\right\}$. Note that since upper- $\Delta$ sets map to lower- $\Delta$ sets, the reverse is true; lower- $\Delta$ sets also map to upper- $\Delta$ sets. Then $\Gamma\left(\delta_{a_{1}, a_{j}}\right)=\delta_{c_{j}, b_{1}}$ implies $\Gamma\left(\Delta_{a_{j}}\right)=\Delta^{c_{j}}$.

To conclude, notice that $\Gamma\left(\delta_{a_{i}, a_{j}}\right) \in \Gamma\left(\Delta^{a_{i}}\right) \cap \Gamma\left(\Delta_{a_{j}}\right)=\Delta_{b_{i}} \cap \Delta^{c_{j}}=\delta_{c_{j}, b_{i}}$. Therefore, every $\delta$ join-irreducible is determined in one of these $n !^{2}$ ways.

Combining Case 1 and Case 2, we have that there are $n !^{2}+n !^{2}=2 \cdot n !^{2}$ ways for an order automorphism of $\operatorname{Res}\left(M_{n}\right)$ to map $\delta$ join-irreducibles to $\delta$ joinirreducibles.

LEMMA 3.6. The values of $\Gamma$ on the $\delta$ join-irreducibles determine $\Gamma$ on the rest of $\operatorname{Res}\left(M_{n}\right)$ for $n \geq 3$.

Proof. There are $n^{2} \delta$ join-irreducibles in $\operatorname{Res}\left(M_{n}\right)$. Suppose $\Gamma \in \operatorname{Aut}\left(\operatorname{Res}\left(M_{n}\right)\right)$ is determined on all of the $\delta$ join-irreducibles. Then, by a quick observation of each type of element in $\operatorname{Res}\left(M_{n}\right)$, we prove that $\Gamma$ is determined on all of $\operatorname{Res}\left(M_{n}\right)$.

Type 1: Because $\Gamma$ is an order automorphism, the bottom and top elements (the all-zero and $\delta_{0,1}$ maps) must remain fixed. Every other $\delta$ map can be written as a join of $\delta$ join-irreducibles, which are determined, and thus every 
Type 1 element is determined. To see this join, see the examples below:

$$
\begin{aligned}
& \Gamma\left(\delta_{0, a_{i}}\right)=\Gamma\left(\delta_{a_{j}, a_{i}} \vee \delta_{a_{k}, a_{i}}\right)=\Gamma\left(\delta_{a_{j}, a_{i}}\right) \vee \Gamma\left(\delta_{a_{k}, a_{i}}\right) \\
& \Gamma\left(\delta_{a_{i}, 1}\right)=\Gamma\left(\delta_{a_{i}, a_{j}} \vee \delta_{a_{i}, a_{k}}\right)=\Gamma\left(\delta_{a_{i}, a_{j}}\right) \vee \Gamma\left(\delta_{a_{i}, a_{k}}\right) .
\end{aligned}
$$

Type 2: The $a_{i}, 1$ maps are the key to our argument. Note first that the maps with two non-1s are the join of two $\delta$ join-irreducibles, and are thus determined. For instance, $\left(a_{1} a_{2} 11 \ldots 1\right)=\delta_{a_{2}, a_{1}} \vee \delta_{a_{1}, a_{2}}$.

Above the maps with two non-1s are elements with one non-1, which are the join of elements with two non-1s and are thus determined. For instance, $\left(a_{1} 1 \ldots 1\right)=\left(a_{1} a_{2} 1 \ldots 1\right) \vee\left(a_{1} a_{3} 1 \ldots 1\right)=\left(\delta_{a_{1}, a_{2}} \vee \delta_{a_{2}, a_{1}}\right) \vee\left(\delta_{a_{2}, a_{1}} \vee \delta_{a_{1}, a_{3}}\right)$

Below the maps with two non-1s are elements with three non-1s. In the $\operatorname{Res}\left(M_{3}\right)$ case, we can now go onto the Type 3 maps, as a map with three non-1s is a permutation map in this instance. In $\operatorname{Res}\left(M_{n}\right)$ for $n>3$, maps with three non-1s are the meet of two elements with two non-1s, and thus are determined by $\Gamma$ since order automorphisms preserve meets. For example, $\left(a_{1} a_{2} a_{3} 11 \ldots 1\right)=\left(1 a_{2} a_{3} 11 \ldots 1\right) \wedge\left(a_{1} 1 a_{3} 11 \ldots 1\right)$.

We keep building downwards; the maps in $\operatorname{Res}\left(M_{n}\right)$ for $n>3$ with four non-1s are the meet of two elements with three non-1s, determined above. So these elements are also determined by $\Gamma$. We continue this process until we arrive at elements that have $(n-1)$ non-1s (i.e. exactly one 1$)$, which are determined by $\Gamma$ as the meet of two elements with $(n-2)$ non-1s (i.e. exactly two 1s).

Type 3: This descending argument brings us to the Type 3 elements, the permutation join-irreducibles. Below an element with $(n-1)$ non-1s is only a single permutation. Since the elements with $(n-1)$ non-1s are determined, hence so are the permutations. For instance, $\left(1 a_{2} a_{3} \ldots a_{n}\right)$ covers only the permutation $\left(a_{1} a_{2} \ldots a_{n}\right)$, and so $\Gamma\left(\left(1 a_{2} a_{3} \ldots a_{n}\right)\right)$ can therefore cover only 
the permutation $\Gamma\left(\left(a_{1} a_{2} \ldots a_{n}\right)\right)$, and hence this permutation is determined.

THEOREM 3.2. Let $f \in \operatorname{Res}\left(M_{n}\right)$ where $n \geq 3$ and $f^{+}$be its residual. Every order automorphism of $\operatorname{Res}\left(M_{n}\right)$ for $n \geq 3$ is induced in one of the following ways:

(i) $\Gamma(f)=\theta \circ f \circ \gamma$ where $\theta, \gamma \in \operatorname{Aut}\left(M_{n}\right)$;

(ii) $\Gamma(f)=\alpha \circ f^{+} \circ \beta$ where $\alpha, \beta$ are dual isomorphisms of $M_{n}$.

Proof. Every element is either a join-irreducible or is the join of join-irreducibles below it. Since order automorphisms preserve joins, they are determined by the values on the join-irreducibles. Lemma 3.5 proves there are $2 n^{2}$ ways for order automorphisms to map the $\delta$ join-irreducibles, and then Lemma 3.6 shows it suffices to know an order automorphism on these $\delta$ join-irreducibles.

Now, we count the left-right composition maps. We construct these maps by composing $\theta \circ f \circ \gamma$ where $\theta, \gamma \in \operatorname{Aut}\left(M_{n}\right), f \in \operatorname{Res}\left(M_{n}\right)$, and also by using $f^{\prime}$ 's residual, $f^{+}$, and composing $\alpha \circ f^{+} \circ \beta$ where $\alpha, \beta$ are dual isomorphisms of $M_{n}$. There are $n$ ! ways to choose $\theta$ and $n$ ! ways to choose $\gamma$, since both are simply permuting the elements $\left\{a_{1}, a_{2}, \ldots, a_{n}\right\}$. This makes for $n !^{2}$ induced maps that use $f \in \operatorname{Res}\left(M_{n}\right)$. There are another $n$ ! ways to choose $\alpha$ and $n$ ! ways to choose $\beta$, yielding another $n !^{2}$ induced maps that use the dual $f^{+}$. Thus, there are $2 n !^{2}$ induced left-right composition maps. Since every induced map is an order automorphism, we have proven that the elements of $\operatorname{Aut}\left(\operatorname{Res}\left(M_{n}\right)\right)$ are all induced by these maps.

Note that, even though our argument is not a constructive one, the two types of induced order automorphisms have a connection between our upper- $\Delta$ and lower- $\Delta$ sets. In Lemma 3.5, we considered two cases for order automorphisms of $\operatorname{Res}\left(M_{n}\right)$. The first one, in which all upper- $\Delta$ sets map to upper- $\Delta$ sets and 
lower- $\Delta$ sets to lower- $\Delta$ sets, corresponds to induced order automorphisms of the form $\theta \circ f \circ \gamma$ from Theorem 3.2. The second case, in which upper- $\Delta$ sets map to lower- $\Delta$ sets and vice versa, corresponds to induced order automorphisms of the form $\alpha \circ f^{+} \circ \beta$ from Theorem 3.2. 


\section{CHAPTER 4 \\ ORDER AUTOMORPHISMS OF RES $\left(N_{5}\right)$}

\subsection{Elements of $\operatorname{Res}\left(N_{k}\right)$}

Before we begin our proof that automorphisms of $\operatorname{Res}\left(N_{5}\right)$ are all induced, let us first explore the size and structure of $\operatorname{Res}\left(N_{k}\right)$ for general $k \geq 5$ where $k$ is the number of elements in the lattice. Refer to Figure 4.1 for the Hasse diagram of $N_{k}$. To determine a residuated map on $N_{k}$, one needs to consider the map's action on its join-irreducibles, i.e. on $\left\{a, b_{1}, b_{2}, \ldots, b_{k-3}\right\}$, and ensure that every join is preserved. For $\operatorname{Res}\left(N_{k}\right)$, this means we must ensure that $f\left(a \vee b_{i}\right)=f\left(a \vee b_{j}\right)=f(1)$ for $1 \leq i, j \leq k-3$ and that $f\left(b_{i}\right) \vee f\left(b_{i+n}\right)=f\left(b_{i+n}\right)$ for $1 \leq i \leq i+n \leq k-3$.

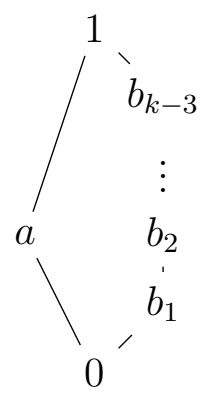

Figure 4.1: $N_{k}$

We will again use slot notation to describe functions in $\operatorname{Res}\left(N_{k}\right)$, using ordered slots for each join-irreducible with $a$ in the first slot and then $b_{1}, b_{2}, \ldots, b_{k-3}$ ordered in the slots afterward. For instance, the function $f=(a 00)$ in $\operatorname{Res}\left(N_{5}\right)$ maps $f(a)=a, f\left(b_{1}\right)=0$, and $f\left(b_{2}\right)=0$. We now discuss the structure of $\operatorname{Res}\left(N_{k}\right)$ 
in a result about its size.

THEOREM 4.1. The number of residuated maps from $N_{k}$ to $N_{k}$ for $k \geq 5$ is given by the formula

$$
k+\left(\begin{array}{c}
2 k-6 \\
k-3
\end{array}\right)+\left(\begin{array}{c}
2 k-5 \\
k-3
\end{array}\right)+\left(\begin{array}{c}
k-1 \\
k-3
\end{array}\right)+\sum_{i=1}^{k-3}\left[\left(\begin{array}{c}
k+i-3 \\
k-3
\end{array}\right)+2 k-i-5\right] .
$$

Proof. We can count the elements of $\operatorname{Res}\left(N_{k}\right)$ by splitting them into four types depending on the image of $a$.

Type 1: $\mathbf{a} \rightarrow \mathbf{0}$ The functions that map a to 0 must map $\left\{b_{1}, b_{2}, \ldots, b_{k-3}\right\}$ to a single element chosen from $N_{k}$ in order to preserve joins. Using the same definition of $\delta$ maps from Chapter 3 , these functions are $\delta_{a, \beta}$ maps where $\beta$ is any element in $N_{k}$. Thus, there are $k$ Type 1 maps since $N_{k}$ contains $k$ elements.

Type 2: a $\rightarrow$ a The functions that fix $a$ are split into two subtypes.

(i) Type 2(i) functions are $\delta$ maps. We have the function $\delta_{0, a}$, which maps every nonzero element to $a$. We also have the functions $\delta_{b_{i}, a}$ (where $1 \leq i \leq k-3)$, which map $\left\{b_{1}, \ldots, b_{i}\right\}$ to 0 and map $\left\{a, b_{i+1}, \ldots, b_{k-3}\right\}$ to $a$. There are $k-3$ choices for $b_{i}$ in the $\delta_{b_{i}, a}$ functions, so then counting $\delta_{0, a}$ as well, there are a total of $k-2$ functions of this subtype.

(ii) Type 2(ii) functions fix $a$ and map $\left\{b_{1}, \ldots, b_{k-3}\right\}$ into $\left\{b_{1}, \ldots, b_{k-3}, 1\right\}$ while preserving order. This is a combination with replacement, with $k-2$ elements from which to choose $k-3$ images. There is no need to concern ourselves with order preservation when counting, because we can simply choose the elements and then put them in the correct order. Hence, using the combination with replacements formula, there are $\left(\begin{array}{c}k-2+k-3-1 \\ k-3\end{array}\right)=\left(\begin{array}{c}2 k-6 \\ k-3\end{array}\right)$ functions of this subtype.

Thus, there are $k-2+\left(\begin{array}{c}2 k-6 \\ k-3\end{array}\right)$ Type 2 functions. 
Type 3: $\mathbf{a} \rightarrow \mathbf{b}_{i}$ The functions that send $a$ to an element $b_{i}$ in the chain are split into three subtypes.

(i) Type 3(i) functions map $a \rightarrow b_{i}$ and then map the chain $\left\{b_{1}, \ldots, b_{k-3}\right\}$ into $\left\{0, b_{1}, \ldots, b_{i}\right\}$ while preserving order. As before, this is a combination with replacement and so for each $i$, there are $\left(\begin{array}{c}i+1+k-3-1 \\ k-3\end{array}\right)=\left(\begin{array}{c}k+i-3 \\ k-3\end{array}\right)$ functions of this subtype.

Note: There are $\delta$ functions hidden in this description; the maps $\delta_{b_{j}, b_{i}}$ map all but some of the $b$-chain to 0 and the remaining nonzero elements to $b_{i}$.

(ii) Type 3(ii) functions map $a \rightarrow b_{i}$ and then map the chain $\left\{b_{1}, \ldots, b_{k-3}\right\}$ into one element from the set $\left\{b_{i+1}, b_{i+2}, \ldots, b_{k-3}\right\}$. Since there are $k-3-i$ choices for an image here, there are $k-3-i$ functions of this subtype for each $i$.

(iii) Type 3(iii) functions map $a \rightarrow b_{i}$ and then map the chain $\left\{b_{1}, \ldots, b_{k-3}\right\}$ to $\{a, 1\}$ while preserving order. To count these, we can simply choose which element in the chain $\left\{b_{1}, \ldots, b_{k-3}\right\}$ is the last to have image $a$. There are $k-3$ ways to choose this, but we also must include the function that sends the chain $\left\{b_{1}, \ldots, b_{k-3}\right\}$ to 1 , so there are a total of $k-2$ functions of this subtype for each $i$.

Thus, there are $\sum_{i=1}^{k-3}\left[\left(\begin{array}{c}k+i-3 \\ k-3\end{array}\right)+2 k-i-5\right]$ Type 3 functions.

Type 4: a $\rightarrow 1$ The functions that send $a$ to 1 are split into two subtypes. Note, however, that these subtypes overlap, so we must remove a double count of some elements.

(i) Type 4(i) functions map $a \rightarrow 1$ and then map the chain $\left\{b_{1}, \ldots, b_{k-3}\right\}$ to $\{0, a, 1\}$ while preserving order. There are $\left(\begin{array}{c}3+k-3-1 \\ k-3\end{array}\right)=\left(\begin{array}{c}k-1 \\ k-3\end{array}\right)$ functions of this subtype. 
(ii) Type 4(ii) functions map $a \rightarrow 1$ and then map the chain $\left\{b_{1}, \ldots, b_{k-3}\right\}$ into $\left\{0, b_{1}, \ldots, b_{k-3}, 1\right\}$ while preserving order. There are $\left(\begin{array}{c}k-1+k-3-1 \\ k-3\end{array}\right)=\left(\begin{array}{c}2 k-5 \\ k-3\end{array}\right)$ functions of this subtype.

Subtype (i) and (ii) of Type 4 functions overlap in the functions that send $a \rightarrow 1$ and then the chain $\left\{b_{1}, \ldots, b_{k-3}\right\}$ to $\{0,1\}$ while preserving order. So we need to subtract the extra copy of these functions that we counted twice. There are $\left(\begin{array}{c}2+k-3-1 \\ k-3\end{array}\right)=\left(\begin{array}{c}k-2 \\ k-3\end{array}\right)=k-2$ of these functions. Thus, there are $\left(\begin{array}{c}k-1 \\ k-3\end{array}\right)+\left(\begin{array}{c}2 k-5 \\ k-3\end{array}\right)-(k-2)$ Type 4 functions.

Noting that these four types of functions include all possible functions that preserve order and joins, and that all four types are disjoint, so we can then count the total number of elements of $\operatorname{Res}\left(N_{k}\right)$ as:

$$
\left|\operatorname{Res}\left(N_{k}\right)\right|=k+\left(\begin{array}{c}
2 k-6 \\
k-3
\end{array}\right)+\left(\begin{array}{c}
2 k-5 \\
k-3
\end{array}\right)+\left(\begin{array}{c}
k-1 \\
k-3
\end{array}\right)+\sum_{i=1}^{k-3}\left[\left(\begin{array}{c}
k+i-3 \\
k-3
\end{array}\right)+2 k-i-5\right] .
$$

If we let $n=k-3$ be the size of the $b$-chain in $N_{k}$, then we can simplify this formula to the following:

$$
\left|\operatorname{Res}\left(N_{k}\right)\right|=2 n(n+1)+3+\left(\begin{array}{c}
2 n \\
n
\end{array}\right)+\left(\begin{array}{c}
2 n+1 \\
n
\end{array}\right)+\left(\begin{array}{c}
n+2 \\
n
\end{array}\right)+\sum_{i=1}^{n}\left[\left(\begin{array}{c}
n+i \\
n
\end{array}\right)-i\right] .
$$

Table 4.1 describes the size of $\operatorname{Res}\left(N_{k}\right)$ for small values of $k$. We have checked The On-Line Encyclopedia of Integer Sequences [12], and it seems that the sequence describing the size of $\operatorname{Res}\left(N_{k}\right)$ is a new sequence.

\subsection{Order Automorphisms of $\operatorname{Res}\left(N_{5}\right)$}

To prove that the order automorphisms of $\operatorname{Res}\left(M_{n}\right)$ are induced, we used a fairly complicated counting argument. Luckily, we can use a much simpler counting argument to prove that the order automorphisms of $\operatorname{Res}\left(N_{5}\right)$ are all induced 
Table 4.1: Size of $\operatorname{Res}\left(N_{k}\right)$

\begin{tabular}{|c|c|}
\hline$k$ & $\left|\operatorname{Res}\left(N_{k}\right)\right|$ \\
\hline \hline 5 & 43 \\
\hline 6 & 120 \\
\hline 7 & 369 \\
\hline 8 & 1244 \\
\hline 9 & 4449 \\
\hline 10 & 16,424 \\
\hline 15 & $13,105,083$ \\
\hline 20 & $>10^{9}$ \\
\hline
\end{tabular}

by left-right compositions. We begin by showing that there are two induced order automorphisms of $\operatorname{Res}\left(N_{5}\right)$, and then show that there are only two order automorphisms of $\operatorname{Res}\left(N_{5}\right)$, and thus we prove that every order automorphism of $\operatorname{Res}\left(N_{5}\right)$ is induced.

LEMMA 4.1. There are two induced order automorphisms of Res $\left(N_{5}\right)$.

Proof. We are counting order automorphisms of the form $\theta \circ f \circ \gamma$ and $\alpha \circ f^{+} \circ \beta$ where $f \in \operatorname{Res}\left(N_{5}\right)$ with residual $f^{+}, \theta, \gamma \in \operatorname{Aut}\left(N_{5}\right)$, and $\alpha, \beta$ are dual isomorphisms of $N_{5}$

Consider $\theta, \gamma \in \operatorname{Aut}\left(N_{5}\right)$. Because of the antisymmetry of $N_{5}, \theta$ and $\gamma$ must both be the identity. So there is only one order automorphism of $\operatorname{Res}\left(N_{5}\right)$ of the form $\theta \circ f \circ \gamma$, and it is the identity.

Consider $\alpha, \beta$ dual isomorphisms of $N_{5}$. The only possibility for such a dual isomorphisms must switch 0 and 1 , switch $b_{1}$ and $b_{2}$, and keep $a$ fixed. Thus $\alpha=\beta$ and there is only one induced order automorphism of the form $\alpha \circ f^{+} \circ \beta$.

LEMMA 4.2. There are only two order automorphisms of $\operatorname{Res}\left(N_{5}\right)$. 
Proof. Clearly, the identity map is an order automorphism of $\operatorname{Res}\left(N_{5}\right)$, and Lemma 4.1 implies there are at least two order automorphisms. So, we are aiming to prove that there is only one non-identity order automorphism. Recall order automorphisms of a lattice map join-irreducibles to join-irreducibles, so to explore the order automorphisms of $\operatorname{Res}\left(N_{5}\right)$, we identified the partially ordered set of join-irreducibles, pictured in Figure 4.2. From Lemma 2.4, we conclude that the $\delta$ join-irreducibles of $\operatorname{Res}\left(N_{5}\right)$ are $\delta_{a, a}, \delta_{a, b_{i}}, \delta_{b_{i}, a}$, and $\delta_{b_{i}, b_{j}}$ where $1 \leq i, j \leq k-3$. Through careful consideration of the elements discussed in Theorem 4.1, we conclude the only other join-irreducible is the map $\left(a b_{1} b_{2}\right)$.

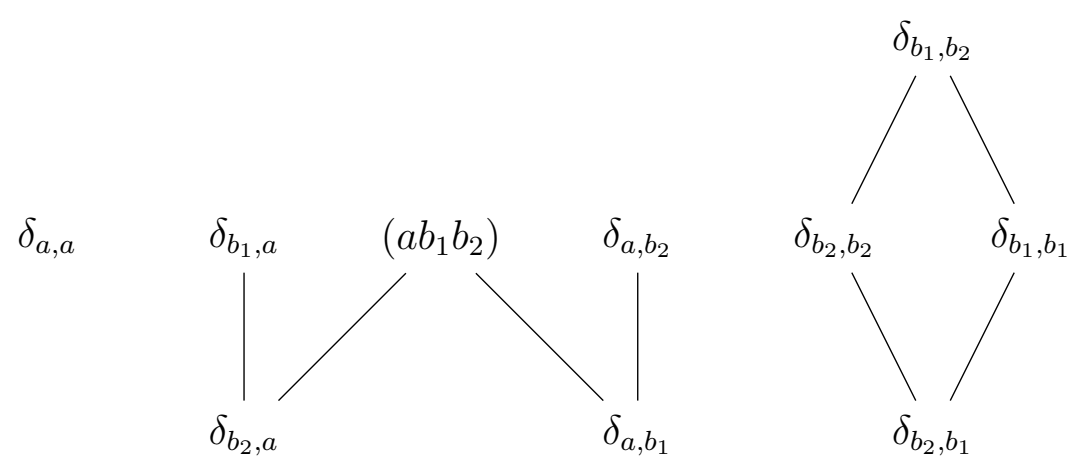

Figure 4.2: Poset of Join-Irreducibles of $\operatorname{Res}\left(N_{5}\right)$

It is easy to see that any order automorphism of $\operatorname{Res}\left(N_{5}\right)$ has only two freedoms: switching $\delta_{b_{2}, b_{2}} \leftrightarrow \delta_{b_{1}, b_{1}}$ and/or switching $\delta_{b_{2}, a} \leftrightarrow \delta_{a, b_{1}}$ (which in turn switches $\left.\delta_{b_{1}, a} \leftrightarrow \delta_{a, b_{2}}\right)$. Our aim is then to show that switching one switches the other, and thus there is only one non-identity order automorphism.

Notice that the only elements covered by (i.e. directly below) $\delta_{b_{2}, 1}$ in $\operatorname{Res}\left(N_{5}\right)$ are the elements $\delta_{b_{2}, a}$ and $\delta_{b_{2}, b_{2}}$. Also, the only elements covered by $\delta_{0, b_{1}}$ are the elements $\delta_{b_{1}, b_{1}}$ and $\delta_{a, b_{1}}$. These are exactly the four elements discussed above whose freedoms we will show are quite restricted. This covering relationship is diagrammed in Figure 4.3, but we must remember there is much more going on in the lattice as 
a whole.
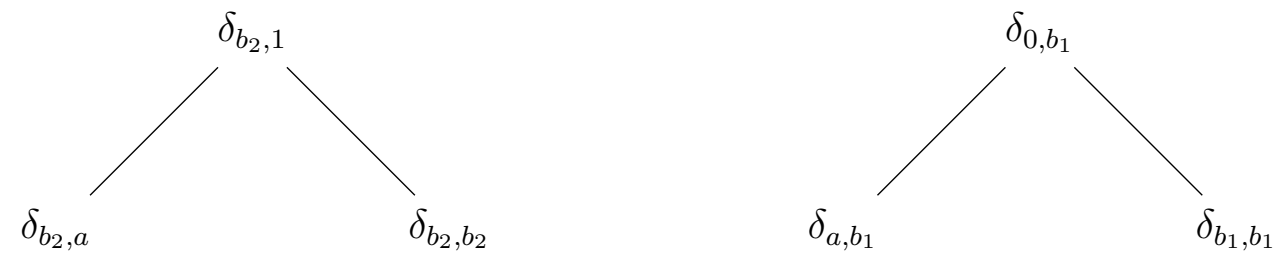

Figure 4.3: Covering relationship of $\delta_{b_{2}, 1}$ and $\delta_{0, b_{1}}$

Now, suppose $\Gamma$ is an order automorphism of $\operatorname{Res}\left(N_{5}\right)$ such that $\Gamma\left(\delta_{b_{2}, b_{2}}\right)=$ $\delta_{b_{1}, b_{1}}$ and $\Gamma\left(\delta_{b_{1}, b_{1}}\right)=\delta_{b_{2}, b_{2}}$. Then since order automorphisms preserve covering relations, and $\delta_{b_{2}, 1}$ covers $\delta_{b_{2}, b_{2}}$, we know that $\Gamma\left(\delta_{b_{2}, 1}\right)$ must be a cover of $\Gamma\left(\delta_{b_{2}, b_{2}}\right)=$ $\delta_{b_{1}, b_{1}}$. Furthermore, the only covers of $\delta_{b_{1}, b_{1}}$ are $\delta_{0, b_{1}}$ and $\delta_{b_{1}, b_{2}}$. Notice, however, that

$$
\Gamma\left(\delta_{b_{1}, b_{2}}\right)=\Gamma\left(\delta_{b_{1}, b_{1}}\right) \vee \Gamma\left(\delta_{b_{2}, b_{2}}\right)=\delta_{b_{2}, b_{2}} \vee \delta_{b_{1}, b_{1}}=\delta_{b_{1}, b_{2}}
$$

Thus, $\delta_{b_{1}, b_{2}}$ remains fixed under $\Gamma$ and so $\Gamma\left(\delta_{b_{2}, 1}\right)=\delta_{0, b_{1}}$ through process of elimination. This, in turn, forces $\Gamma\left(\delta_{b_{2}, a}\right)=\delta_{a, b_{1}}$ and $\Gamma\left(\delta_{a, b_{1}}\right)=\delta_{b_{2}, a}$ because of the covering relation of Figure 4.3.

So, if an order automorphism switches $\delta_{b_{2}, b_{2}} \leftrightarrow \delta_{b_{1}, b_{1}}$, then it also switches $\delta_{b_{2}, a} \leftrightarrow \delta_{a, b_{1}}$ (which in turn switches $\delta_{b_{1}, a} \leftrightarrow \delta_{a, b_{2}}$ ). Note that, had we begun instead with $\Gamma\left(\delta_{b_{2}, a}\right)=\delta_{a, b_{1}}$, a similar process would have shown that $\Gamma\left(\delta_{b_{2}, b_{2}}\right)=\delta_{b_{1}, b_{1}}$. We can therefore conclude that there is only one non-identity order automorphism of $\operatorname{Res}\left(N_{5}\right)$.

The proof of the following theorem is a direct result of Lemmas 4.1 and 4.2.

THEOREM 4.2. Let $f \in \operatorname{Res}\left(N_{5}\right)$ and $f^{+}$be its residual. There are only two order automorphisms of Res $\left(N_{5}\right)$ and they are both induced by left-right composition. Their characterizations are below. 
(i) $\Gamma(f)=\theta \circ f \circ \theta$ where $\theta=i d \in \operatorname{Aut}\left(N_{5}\right)$ is the identity order automorphism;

(ii) $\Gamma(f)=\alpha \circ f^{+} \circ \alpha$, where $\alpha$ is the only dual isomorphism of $N_{5}$, is the only non-identity order automorphism.

To this point, we are unable to extend these results to $\operatorname{Res}\left(N_{k}\right)$ for general $k$. However, we have accomplished much of the goal of this dissertation. We wanted to study the order automorphisms of $\operatorname{Res}(L)$ where $L$ is nondistributive and their connection to some known induced order automorphisms. The canonical nondistributive lattices are $M_{3}$ and $N_{5}$, and we have proved that order automorphisms of $\operatorname{Res}\left(M_{3}\right)$ and $\operatorname{Res}\left(N_{5}\right)$ are indeed all of the induced type. Of course, we would like a result for any nondistributive lattice $L$, which would contain one or both of $M_{3}$ or $N_{5}$ as a sublattice.

Most of our explorations so far have been in hopes of finding a larger pattern in which to extrapolate more abstract ideas about $\operatorname{Res}(L)$ for $L$ a finite, nondistributive lattice. However, extension of our results has proven difficult. Determining, in general, the structure of $\operatorname{Res}(L)$ is a complex task. In the distributive case [18], some non-induced order automorphisms were found when considering products of lattices. Hoping for a similar result, we began a study of $\operatorname{Res}\left(M_{3} \times 2\right)$; our findings are discussed in the next chapter. 


\section{CHAPTER 5 \\ ORDER AUTOMORPHISMS OF RES $\left(M_{3} \times 2\right)$}

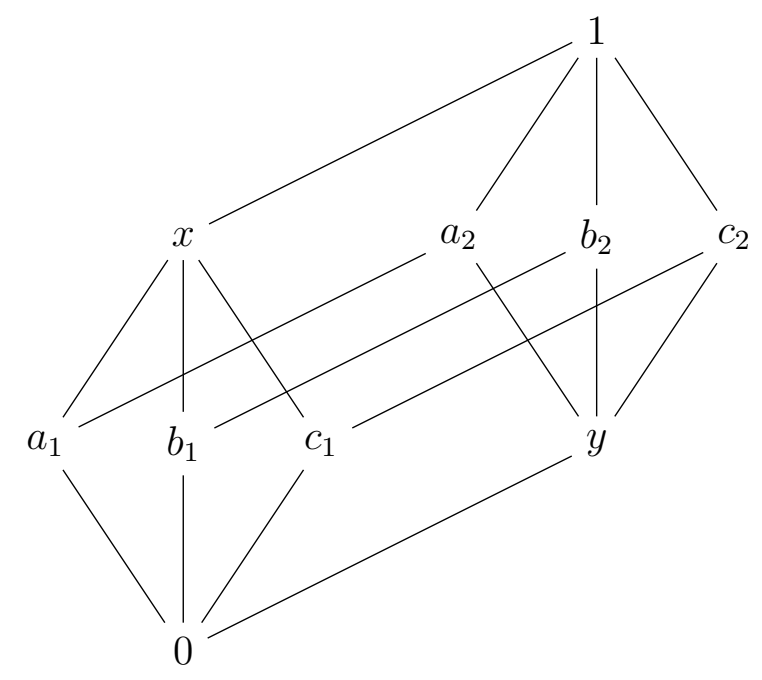

Figure 5.1: $M_{3} \times 2$

After proving (unexpectedly) that order automorphisms of $\operatorname{Res}\left(M_{3}\right)$ and $\operatorname{Res}\left(N_{5}\right)$ are all induced, we began searching for an example of nondistributive $L$ for which the order automorphisms of $\operatorname{Res}(L)$ are not all induced. In Lagani's [18] results, he found examples of such when considering products of lattices, so we began an investigation of $M_{3} \times 2$, whose Hasse diagram is found in Figure 5.1. To this point, we have not been able to prove whether or not every order automorphism of $\operatorname{Res}\left(M_{3} \times 2\right)$ is induced. We will prove, however, that order automorphisms that send $\delta$ maps to $\delta$ maps are indeed all induced. In this chapter, we will provide a description of $\operatorname{Res}\left(M_{3} \times 2\right)$ and discuss what we know so far about its order automorphisms. 


\subsection{Structure of $\operatorname{Res}\left(M_{3} \times 2\right)$}

When building maps in $\operatorname{Res}\left(M_{3} \times 2\right)$, we need only to concern ourselves with the images of the join-irreducible elements $\left\{a_{1}, b_{1}, c_{1}, y\right\}$ since every element of a lattice is a (not necessarily unique) join of join-irreducibles. Since residuated maps are equivalent to join-homomorphisms, it seems at first as if we have four conditions to check:

$$
\begin{gathered}
a_{1} \vee b_{1} \stackrel{(1)}{=} a_{1} \vee c_{1} \stackrel{(2)}{=} b_{1} \vee c_{1} \\
a_{1} \vee b_{1} \vee y \stackrel{(3)}{=} a_{1} \vee c_{1} \vee y \stackrel{(4)}{=} b_{1} \vee c_{1} \vee y .
\end{gathered}
$$

However, one may quickly notice that once conditions (1) and (2) are met, conditions (3) and (4) come naturally since we are merely joining $y$. Thus, we may build our residuated maps by ensuring that the maps are join preserving in our first (bottom) copy of $M_{3}$ while allowing the image of $y$ to be any of the 10 elements in $M_{3} \times 2$. Effectively, this allows us to quickly build several copies of $\operatorname{Res}\left(M_{3}\right)$ as sublattices of $\operatorname{Res}\left(M_{3} \times 2\right)$. To do so, we merely extend an element $f$ of $\operatorname{Res}\left(M_{3}\right)$ to $\operatorname{Res}\left(M_{3} \times 2\right)$ by mapping $y$ to a fixed element in $M_{3} \times 2$. This gives us 10 copies of $\operatorname{Res}\left(M_{3}\right)$, and thus the lattice $\operatorname{Res}\left(M_{3}\right) \times\left(M_{3} \times 2\right)$ as a sublattice of $\operatorname{Res}\left(M_{3} \times 2\right)$. The remaining elements of $\operatorname{Res}\left(M_{3} \times 2\right)$ are quickly found since we have only two conditions to check. A full list of the 2500 elements of $\operatorname{Res}\left(M_{3} \times 2\right)$ is found in Table 5.1.

\subsection{Join-Irreducible Elements of $\operatorname{Res}\left(M_{3} \times 2\right)$}

We have shown that the structure of $\operatorname{Res}\left(M_{3} \times 2\right)$ is very similar to that of $\operatorname{Res}\left(M_{3}\right)$. By a careful investigation of the chart of elements of $\operatorname{Res}\left(M_{3} \times 2\right)$, we can see that there are 22 join-irreducible elements, and like in $\operatorname{Res}\left(M_{3}\right)$, they are split into $\delta$ join-irreducibles and the permutation join-irreducibles. Lemma 2.4 tells us that the $\delta$ join-irreducible elements of $\operatorname{Res}\left(M_{3} \times 2\right)$ are of the form $\delta_{\alpha, \beta}$ 


\begin{tabular}{|c|c|c|c|c|}
\hline$f\left(\alpha_{1}\right)$ & $f\left(\beta_{1}\right)$ & $f\left(\gamma_{1}\right)$ & $f(y)$ & Count \\
\hline 0 & $\mu$ & $\mu$ & any & 270 \\
\hline$\mu$ & $\mu$ & $\mu$ & any & 100 \\
\hline$\alpha_{1}$ & $\beta_{1}$ & $\gamma_{1}$ & any & 60 \\
\hline$\alpha_{1}$ & $\beta_{1}$ & $\mathrm{x}$ & any & 180 \\
\hline$\alpha_{1}$ & $\mathrm{x}$ & $\mathrm{x}$ & any & 90 \\
\hline$\alpha_{1}$ & $\alpha_{2}$ & $\alpha_{2}$ & any & 90 \\
\hline$\alpha_{1}$ & $\alpha_{2}$ & $\mathrm{y}$ & any & 180 \\
\hline$\alpha_{1}$ & $\beta_{2}$ & $\gamma_{2}$ & any & 180 \\
\hline$\alpha_{2}$ & $\alpha_{2}$ & $\mathrm{y}$ & any & 90 \\
\hline$\alpha_{1}$ & $\beta_{2}$ & 1 & any & 360 \\
\hline$\alpha_{2}$ & $\beta_{2}$ & $\gamma_{2}$ & any & 60 \\
\hline$\alpha_{2}$ & $\beta_{2}$ & $\mathrm{x}$ & any & 180 \\
\hline$\alpha_{2}$ & $\beta_{2}$ & 1 & any & 180 \\
\hline$\alpha_{2}$ & $\mathrm{x}$ & 1 & any & 180 \\
\hline $\mathrm{x}$ & $\mathrm{y}$ & 1 & any & 60 \\
\hline$\mu$ & 1 & 1 & any & 240 \\
\hline & & & & 2500 \\
\hline
\end{tabular}

Table 5.1: $\operatorname{Res}\left(M_{3} \times 2\right)$

where $\mu$ is arbitrary element of $M_{3} \times 2$ and $\alpha_{1}, \beta_{1}, \gamma_{1}$ are arbitrary but distinct elements from $\left\{a_{1}, b_{1}, c_{1}\right\}$ with $\alpha_{2}, \beta_{2}, \gamma_{2}$ as their corresponding covers. 
where $\alpha \in\left\{a_{2}, b_{2}, c_{2}, x\right\}$ and $\beta \in\left\{a_{1}, b_{1}, c_{1}, y\right\}$. The permutation join-irreducible elements are formed by permuting the elements $\left\{a_{1}, b_{1}, c_{1}\right\}$ and mapping $y$ to 0 . To list them in the "slot notation" as we did in $\operatorname{Res}\left(M_{3}\right)$, we have to consider the slots $\left(f\left(a_{1}\right) f\left(b_{1}\right) f\left(c_{1}\right) \mid f(y)\right)$, and we have the permutation join-irreducibles listed below:

$$
\left(a_{1} b_{1} c_{1} \mid 0\right),\left(a_{1} c_{1} b_{1} \mid 0\right),\left(b_{1} a_{1} c_{1} \mid 0\right),\left(b_{1} c_{1} a_{1} \mid 0\right),\left(c_{1} a_{1} b_{1} \mid 0\right),\left(c_{1} b_{1} a_{1} \mid 0\right)
$$

We can gather several important facts about the join-irreducibles of $\operatorname{Res}\left(M_{3} \times\right.$ 2 ) by simply looking at their covers. The $\delta$ join-irreducibles of the form $\delta_{\alpha_{2}, \beta_{1}}$ where $\alpha_{2} \in\left\{a_{2}, b_{2}, c_{2}\right\}$ and $\beta_{1} \in\left\{a_{1}, b_{1}, c_{1}\right\}$ have 13 covers. For example, covers of $\delta_{a_{2}, b_{1}}=\left(0 b_{1} b_{1} \mid 0\right)$ are listed below:

$$
\begin{array}{rlrl}
\left(0 b_{1} b_{1} \mid b_{1}\right) & =\delta_{b_{1}, b_{1}} & \left(0 b_{1} b_{1} \mid a_{1}\right) & \left(a_{1} b_{1} x \mid 0\right) \\
\left(b_{1} b_{1} b_{1} \mid 0\right) & =\delta_{y, b_{1}} & \left(0 b_{1} b_{1} \mid c_{1}\right) & \left(c_{1} x b_{1} \mid 0\right) \\
\left(0 b_{2} b_{2} \mid 0\right) & =\delta_{a_{2}, b_{2}} & \left(0 b_{1} b_{1} \mid y\right) & \left(c_{1} b_{1} x \mid 0\right) \\
(0 x x \mid 0) & =\delta_{a_{2}, x} & \left(a_{1} x b_{1} \mid 0\right) & \left(y b_{1} b_{2} \mid 0\right) \\
& & \left(y b_{2} b_{1} \mid 0\right)
\end{array}
$$

The $\delta$ join-irreducibles of the form $\delta_{x, \beta_{1}}$ have 20 covers. For example, the covers of $\delta_{x, a_{1}}=\left(000 \mid a_{1}\right)$ are listed below:

$\begin{aligned}\left(000 \mid a_{2}\right) & =\delta_{x, a_{2}} & \left(0 b_{1} b_{1} \mid a_{1}\right) & \left(c_{1} c_{1} 0 \mid a_{1}\right) & \left(a_{1} c_{1} b_{1} \mid a_{1}\right) \\ (000 \mid x) & =\delta_{x, x} & \left(b_{1} 0 b_{1} \mid a_{1}\right) & \left(0 y y \mid a_{1}\right) & \left(b_{1} a_{1} c_{1} \mid a_{1}\right) \\ \left(0 a_{1} a_{1} \mid a_{1}\right) & =\delta_{a_{1}, a_{1}} & \left(b_{1} b_{1} 0 \mid a_{1}\right) & \left(y 0 y \mid a_{1}\right) & \left(b_{1} c_{1} a_{1} \mid a_{1}\right) \\ \left(a_{1} 0 a_{1} \mid a_{1}\right) & =\delta_{b_{1}, a_{1}} & \left(0 c_{1} c_{1} \mid a_{1}\right) & \left(y y 0 \mid a_{1}\right) & \left(c_{1} a_{1} b_{1} \mid a_{1}\right) \\ \left(a_{1} a_{1} 0 \mid a_{1}\right) & =\delta_{c_{1}, a_{1}} & \left(c_{1} 0 c_{1} \mid a_{1}\right) & \left(a_{1} b_{1} c_{1} \mid a_{1}\right) & \left(c_{1} b_{1} a_{1} \mid a_{1}\right)\end{aligned}$

The $\delta$ join-irreducibles of the form $\delta_{\alpha_{2}, y}$ have 20 covers also. For example, 
the covers of $\delta_{a_{2}, y}=(0 y y \mid 0)$ are listed below:

$\begin{aligned}(0 y y \mid y) & =\delta_{a_{1}, y} & \left(0 y y \mid a_{1}\right) & \left(b_{1} b_{2} y \mid 0\right) & \left(a_{1} c_{2} b_{2} \mid 0\right) \\ (\text { yyy } \mid 0) & =\delta_{y, y} & \left(0 y y \mid b_{1}\right) & \left(b_{1} y b_{2} \mid 0\right) & \left(b_{1} a_{2} c_{2} \mid 0\right) \\ \left(0 a_{2} a_{2} \mid 0\right) & =\delta_{a_{2}, a_{2}} & \left(0 y y \mid c_{1}\right) & \left(c_{1} c_{2} y \mid 0\right) & \left(b_{1} c_{2} a_{2} \mid 0\right) \\ \left(0 b_{2} b_{2} \mid 0\right) & =\delta_{a_{2}, b_{2}} & \left(a_{1} a_{2} y \mid 0\right) & \left(c_{1} y c_{2} \mid y\right) & \left(c_{1} a_{2} b_{2} \mid 0\right) \\ \left(0 c_{2} c_{2} \mid 0\right) & =\delta_{a_{2}, c_{2}} & \left(a_{1} y a_{2} \mid 0\right) & \left(a_{1} b_{2} c_{2} \mid 0\right. & \left(c_{1} b_{2} a_{2} \mid 0\right)\end{aligned}$

The only remaining $\delta$ join-irreducible left is $\delta_{x, y=(000 \mid y)}$, which has 21 covers:

$\begin{array}{llll}\left(000 \mid a_{2}\right)=\delta_{x, a_{2}} & \left(a_{1} a_{1} 0 \mid y\right) & \left(0 c_{1} c_{1} \mid y\right) & \left(a_{1} b_{1} c_{1} \mid y\right) \\ \left(000 \mid b_{2}\right)=\delta_{x, b_{2}} & \left(0 b_{1} b_{1} \mid y\right) & \left(c_{1} 0 c_{1} \mid y\right) & \left(a_{1} c_{1} b_{1} \mid y\right) \\ \left(000 \mid c_{2}\right)=\delta_{x, c_{2}} & \left(b_{1} 0 b_{1} \mid y\right) & (0 y y \mid y) & \left(b_{1} a_{1} c_{1} \mid y\right) \\ \left(0 a_{1} a_{1} \mid y\right) & \left(b_{1} b_{1} 0 \mid y\right) & (y 0 y \mid y) & \left(b_{1} c_{1} a_{1} \mid y\right) \\ \left(a_{1} 0 a_{1} \mid y\right) & \left(c_{1} c_{1} 0 \mid y\right) & (y y 0 \mid y) & \left(c_{1} a_{1} b_{1} \mid y\right) \\ & & \left(c_{1} b_{1} a_{1} \mid y\right)\end{array}$

Finally, the permutation join-irreducibles each have 10 covers. For instance, the covers of $\left(a_{1} b_{1} c_{1} \mid 0\right)$ are:

$$
\begin{array}{ll}
\left(a_{1} b_{1} c_{1} \mid a_{1}\right) & \left(a_{1} x c_{1} \mid 0\right) \\
\left(a_{1} b_{1} c_{1} \mid b_{1}\right) & \left(x b_{1} c_{1} \mid 0\right) \\
\left(a_{1} b_{1} c_{1} \mid c_{1}\right) & \left(a_{1} b_{2} c_{2} \mid 0\right) \\
\left(a_{1} b_{1} c_{1} \mid y\right) & \left(a_{2} b_{1} c_{2} \mid 0\right) \\
\left(a_{1} b_{1} x \mid 0\right) & \left(a_{2} b_{2} c_{1} \mid 0\right)
\end{array}
$$

We can immediately draw three conclusions from this discussion of the covers of each join-irreducible in $\operatorname{Res}\left(M_{3} \times 2\right)$. 
1. Since $\delta \mathrm{s}$ of the form similar to $\delta_{a_{2}, b_{1}}$ are the only join-irreducibles with 13 covers, we conclude that an order automorphism of $\operatorname{Res}\left(M_{3} \times 2\right)$ must keep these special $\delta$ s invariant as a set. In other words, if $\Gamma \in \operatorname{Aut}\left(\operatorname{Res}\left(M_{3} \times 2\right)\right)$, then $\Gamma\left(\delta_{\alpha_{2}, \beta_{1}}\right)=\delta_{\gamma_{2}, \theta_{1}}$ where $\alpha_{2}, \gamma_{2} \in\left\{a_{2}, b_{2}, c_{2}\right\}$ and $\beta_{1}, \theta_{1} \in\left\{a_{1}, b_{1}, c_{1}\right\}$.

2. The $\delta \mathrm{s}$ of the form similar to $\delta_{x, a_{1}}$ and those of the form similar to $\delta_{a_{2}, y}$ each have 20 covers, while permutation join-irreducibles only have 10. So, it seems that an order automorphism can switch elements such as $\delta_{x, a_{1}}$ and $\delta_{a_{2}, y}$, but it cannot map any $\delta$ join-irreducible to a permutation join-irreducible. So it must be the case that an order automorphism sends permutation join-irreducibles to permutation join-irreducibles and $\delta$ join-irreducibles to $\delta$ join-irreducibles.

3. The join-irreducible $\delta_{x, y}$ is the only join-irreducible with 21 covers, so an order automorphism of $\operatorname{Res}\left(M_{3} \times 2\right)$ must fix $\delta_{x, y}$.

To further discuss the $\delta$ join-irreducibles neatly and concisely, we will again define upper- $\Delta$ and lower- $\Delta$ sets. We are again going to define these sets using the " $M_{3}$ property." Recall a set is defined with this property when it is a three-element set for which the join of any two elements is the same. We will define our $\Delta$ sets in such a way that they will be the only sets containing three $\delta$ join-irreducibles with the " $M_{3}$ property". First, let us define the sets, and then we will explain why these sets are the only to hold such a characteristic. Let the upper- $\Delta$ and lower- $\Delta$ sets be defined as below:

$$
\begin{array}{ll}
\Delta^{a_{2}}=\left\{\delta_{a_{2}, a_{1}}, \delta_{a_{2}, b_{1}}, \delta_{a_{2}, c_{1}}\right\} & \Delta_{a_{1}}=\left\{\delta_{a_{2}, a_{1}}, \delta_{b_{2}, a_{1}}, \delta_{c_{2}, a_{1}}\right\} \\
\Delta^{b_{2}}=\left\{\delta_{b_{2}, a_{1}}, \delta_{b_{2}, b_{1}}, \delta_{b_{2}, c_{1}}\right\} & \Delta_{b_{1}}=\left\{\delta_{a_{2}, b_{1}}, \delta_{b_{2}, b_{1}}, \delta_{c_{2}, b_{1}}\right\} \\
\Delta^{c_{2}}=\left\{\delta_{c_{2}, a_{1}}, \delta_{c_{2}, b_{1}}, \delta_{c_{2}, c_{1}}\right\} & \Delta_{c_{1}}=\left\{\delta_{a_{2}, c_{1}}, \delta_{b_{2}, c_{1}}, \delta_{c_{2}, b_{1}}\right\} \\
\Delta^{x}=\left\{\delta_{x, a_{1}}, \delta_{x, b_{1}}, \delta_{x, c_{1}}\right\} & \Delta_{y}=\left\{\delta_{a_{2}, y}, \delta_{b_{2}, y}, \delta_{c_{2}, y}\right\}
\end{array}
$$

Notice the sets are defined slightly different than in our discussion of $\operatorname{Res}\left(M_{3}\right)$. 
For instance, the set $\Delta^{a_{2}}$ does not contain every $\delta$ join-irreducible with first index $a_{2}$ since we leave out the element $\delta_{a_{2}, y}$. This is similar for every upper- $\Delta$ set, with the reason being that the addition of such an element would break the $M_{3}$ property. Similarly, notice that the lower- $\Delta$ sets leave out elements such as $\delta_{x, a_{1}}$. Again, the addition of these elements would break the $M_{3}$ property. The join-irreducible $\delta_{x, y}$ has been left out of our $\Delta$ sets. This is because adding it to either $\Delta^{x}$ or $\Delta_{y}$ will break our $M_{3}$ property, and we have already discussed that $\delta_{x, y}$ must be fixed, so it has no real bearing on our argument.

We can easily show that the sets above are the only sets containing three $\delta$ join-irreducibles and having the $M_{3}$ property. Suppose we have distinct $\alpha, \beta \in$ $\left\{a_{2}, b_{2}, c_{2}, x\right\}$ and distinct $\gamma, \theta \in\left\{a_{1}, b_{1}, c_{1}, y\right\}$, then we have the following join:

$$
\left(\delta_{\alpha, \gamma} \vee \delta_{\beta, \theta}\right)(z)= \begin{cases}0 & z \leq \alpha, z \leq \beta \\ \gamma & z \not \leq \alpha, z \leq \beta \\ \theta & z \leq \alpha, z \not \leq \beta \\ \gamma \vee \theta & z \not \leq \alpha, z \not \leq \beta\end{cases}
$$

It is clear that the only $\delta$ join-irreducibles below this map are the two that joined to form it, and thus no third $\delta$ join-irreducible can be found to make a set with the $M_{3}$ property.

Since these are the only sets of $\delta$ join-irreducibles we can form with the $M_{3}$ property, we know any order automorphism of $\operatorname{Res}\left(M_{3} \times 2\right)$ keeps these sets invariant upon one another. Furthermore, from our discussion of covers, we know an order automorphisms of $\operatorname{Res}\left(M_{3} \times 2\right)$ maps the sets $\Delta^{a_{2}}, \Delta^{b_{2}}, \Delta^{c_{2},}, \Delta_{a_{1}}, \Delta_{b_{1}}, \Delta_{c_{1}}$ only the one another and we will show that an order automorphism maps $\Delta^{x}$ and $\Delta_{y}$ only to one another. 
5.3 Order Automorphisms of $\operatorname{Res}\left(M_{3} \times 2\right)$, with an assumption

Now the argument proceeds very similarly to the argument for $\operatorname{Res}\left(M_{3}\right)$. We can show among $\Delta^{a_{2}}, \Delta^{b_{2}}, \Delta^{c_{2}}, \Delta_{a_{1}}, \Delta_{b_{1}}, \Delta_{c_{1}}$ that either uppers $\leftrightarrow$ uppers or uppers $\leftrightarrow$ lowers. Then, we must make an assumption that an order automorphism of $\operatorname{Res}\left(M_{3} \times 2\right)$ sends every $\delta$ function to a $\delta$ function. With this rather strong assumption, we then can show that once these sets are determined by an order automorphism, then so are $\Delta^{x}$ and $\Delta_{y}$ (Lemma 5.4). Finally, we can show that this is enough information to also determine the permutation join-irreducibles, and thus the entire order automorphism. Just like with $\operatorname{Res}\left(M_{3}\right)$, there are 72 order automorphisms found by determining $\Delta^{a_{2}}, \Delta^{b_{2}}, \Delta^{c_{2}}, \Delta_{a_{1}}, \Delta_{b_{1}}, \Delta_{c_{1}}$, and there are 72 induced order automorphisms, so every order automorphism is induced (given $\delta$ s go to $\delta \mathrm{s})$.

LEMMA 5.1. Suppose $\Gamma \in \operatorname{Aut}\left(\operatorname{Res}\left(M_{3} \times 2\right)\right)$ such that $\Gamma\left(\Delta^{u_{2}}\right)=\Delta^{v_{2}}$. for some $u_{2}, v_{2} \in\left\{a_{2}, b_{2}, c_{2}\right\}$. Then $\Gamma\left(\left\{\Delta^{a_{2}}, \Delta^{b_{2}}, \Delta^{c_{2}}\right\}\right)=\left\{\Delta^{a_{2}}, \Delta^{b_{2}}, \Delta^{c_{2}}\right\}$ and $\Gamma\left(\left\{\Delta_{a_{1}}, \Delta_{b_{1}}, \Delta_{c_{1}}\right\}\right)=$ $\left\{\Delta_{a_{1}}, \Delta_{b_{1}}, \Delta_{c_{1}}\right\}$.

Proof. Suppose $\Gamma\left(\Delta^{u_{2}}\right)=\Delta^{v_{2}}$ such that

$$
\begin{aligned}
& \Gamma\left(\delta_{u_{2}, a_{1}}\right)=\delta_{v_{2}, r_{1}} \\
& \Gamma\left(\delta_{u_{2}, b_{1}}\right)=\delta_{v_{2}, s_{1}} \\
& \Gamma\left(\delta_{u_{2}, c_{1}}\right)=\delta_{v_{2}, t_{1}}
\end{aligned}
$$

where $\left\{r_{1}, s_{1}, t_{1}\right\}=\left\{a_{1}, b_{1}, c_{1}\right\}$. Now consider $\Gamma\left(\Delta_{a_{1}}\right)$ by looking at the image of $\delta_{u_{2}, a_{1}}$. We have that $\Gamma\left(\delta_{u_{2}, a_{1}}\right)=\delta_{v_{2}, r_{1}} \in \Delta^{v_{2}} \cap \Delta_{r_{1}}$, and thus, since $\Delta^{v_{2}}$ is already an image under $\Gamma$, it must be that $\Gamma\left(\Delta_{a_{1}}\right)=\Delta_{r_{1}}$. Similarly, $\Gamma\left(\delta_{u_{2}, b_{1}}\right)=\delta_{v_{2}, s_{1}}$, and thus $\Gamma\left(\Delta_{b_{1}}\right)=\Delta_{s_{1}}$. Finally, $\Gamma\left(\delta_{u_{2}, c_{1}}\right)=\delta_{v_{2}, t_{1}}$, and thus $\Gamma\left(\Delta_{c_{1}}\right)=\Delta_{t_{1}}$. This proves that the sets $\Delta_{a_{1}}, \Delta_{b_{1}}$, and $\Delta_{c_{1}}$ are mapped to one another, leaving the sets $\Delta^{a_{2}}, \Delta^{b_{2}}$, and $\Delta^{c_{2}}$ to map to one another. 
LEMMA 5.2. Suppose $\Gamma \in \operatorname{Aut}\left(\operatorname{Res}\left(M_{3} \times 2\right)\right)$ such that $\Gamma\left(\Delta^{u_{2}}\right)=\Delta_{v_{1}}$ for some $u_{2} \in$ $\left\{a_{2}, b_{2}, c_{2}\right\}$ and some $v_{1} \in\left\{a_{1}, b_{1}, c_{1}\right\}$. Then $\Gamma\left(\left\{\Delta^{a_{2}}, \Delta^{b_{2}}, \Delta^{c_{2}}\right\}\right)=\left\{\Delta_{a_{1}}, \Delta_{b_{1}}, \Delta_{c_{1}}\right\}$ and $\Gamma\left(\left\{\Delta_{a_{1}}, \Delta_{b_{1}}, \Delta_{c_{1}}\right\}\right)=\left\{\Delta^{a_{2}}, \Delta^{b_{2}}, \Delta^{c_{2}}\right\}$.

Proof. Suppose $\Gamma\left(\Delta^{u_{2}}\right)=\Delta_{v_{1}}$ such that

$$
\begin{aligned}
& \Gamma\left(\delta_{u_{2}, a_{1}}\right)=\delta_{r_{2}, v_{1}} \\
& \Gamma\left(\delta_{u_{2}, b_{1}}\right)=\delta_{s_{2}, v_{1}} \\
& \Gamma\left(\delta_{u_{2}, c_{1}}\right)=\delta_{t_{2}, v_{1}}
\end{aligned}
$$

where $\left\{r_{2}, s_{2}, t_{2}\right\}=\left\{a_{2}, b_{2}, c_{2}\right\}$. Now consider $\Gamma\left(\Delta_{a_{1}}\right)$ by looking at the image of $\delta_{u_{2}, a_{1}}$. We have that $\Gamma\left(\delta_{u_{2}, a_{1}}\right)=\delta_{r_{2}, v_{1}} \in \Delta^{r_{2}} \cap \Delta_{v_{1}}$, and thus, since $\Delta_{v_{1}}$ is already an image under $\Gamma$, we have $\Gamma\left(\Delta_{a_{1}}\right)=\Delta^{r_{2}}$. Similarly, $\Gamma\left(\Delta_{b_{1}}\right)=\Delta^{s_{2}}$ and $\Gamma\left(\Delta_{c_{1}}\right)=\Delta^{t_{2}}$. Thus, the sets $\Delta_{a_{1}}, \Delta_{b_{1}}, \Delta_{c_{1}}$ are mapped to $\Delta^{a_{2}}, \Delta^{b_{2}}$, and $\Delta^{c_{2}}$, leaving $\Delta^{a_{2}}, \Delta^{b_{2}}, \Delta^{c_{2}}$ to map to $\Delta_{a_{1}}, \Delta_{b_{1}}$, and $\Delta_{c_{1}}$.

LEMMA 5.3. There are 72 ways for order automorphisms of Res $\left(M_{3} \times 2\right)$ to map the sets $\Delta^{a_{2}}, \Delta^{b_{2}}, \Delta^{c_{2}}, \Delta_{a_{1}}, \Delta_{b_{1}}, \Delta_{c_{1}}$.

Proof. We have discussed already that an order automorphism of $\operatorname{Res}\left(M_{3} \times 2\right)$ must map these six $\Delta$ sets to one another. Lemma 5.1 and Lemma 5.2 imply that either the three upper- $\Delta \mathrm{s}$ map to upper- $\Delta \mathrm{s}$ or the three upper- $\Delta \mathrm{s}$ map to the three lower- $\Delta \mathrm{s}$, so we consider two cases.

Case 1: Consider automorphisms that map $\Delta^{a_{2}}, \Delta^{b_{2}}$, and $\Delta^{c_{2}}$ among themselves. We will show that specifying $\Gamma \in \operatorname{Aut}\left(\operatorname{Res}\left(M_{3} \times 2\right)\right)$ on one of $\Delta^{a_{2}}, \Delta^{b_{2}}, \Delta^{c_{2}}$ determines $\Gamma$ on the remaining five $\Delta$ sets. There are $3 !=6$ ways to choose to map $\Delta^{a_{2}}, \Delta^{b_{2}}, \Delta^{c_{2}}$ onto one another and then $3 !=6$ ways to specify $\Gamma$ on just one of these sets, making for a total of $3 !^{2}=36$ automorphisms that map these upper- $\Delta \mathrm{s}$ to upper- $\Delta \mathrm{s}$. 
Without loss of generality, suppose $\Gamma \in \operatorname{Aut}\left(\operatorname{Res}\left(M_{3} \times 2\right)\right)$ such that $\Gamma\left(\Delta^{a_{2}}\right)=$ $\Delta^{u_{2}}, \Gamma\left(\Delta^{b_{2}}\right)=\Delta^{v_{2}}$, and $\Gamma\left(\Delta^{c_{2}}\right)=\Delta^{w_{2}}$ where $\left\{u_{2}, v_{2}, w_{2}\right\}=\left\{a_{2}, b_{2}, c_{2}\right\}$. Suppose we specify $\Gamma$ on $\Delta^{a_{2}}$ such that

$$
\begin{aligned}
& \Gamma\left(\delta_{a_{2}, a_{1}}\right)=\delta_{u_{2}, r_{1}} \\
& \Gamma\left(\delta_{a_{2}, b_{1}}\right)=\delta_{u_{2}, s_{1}} \\
& \Gamma\left(\delta_{a_{2}, c_{1}}\right)=\delta_{u_{2}, t_{1}}
\end{aligned}
$$

where $\left\{r_{1}, s_{1}, t_{1}\right\}=\left\{a_{1}, b_{1}, c_{1}\right\}$.

Note that lower- $\Delta$ sets map to other lower- $\Delta$ sets in this case. So we have, $\Gamma\left(\delta_{a_{2}, a_{1}}\right)=\delta_{u_{2}, r_{1}}$ implies $\Gamma\left(\Delta_{a_{1}}\right)=\Delta_{r_{1}}$. Similarly, $\Gamma\left(\Delta_{b_{1}}\right)=\Delta_{s_{1}}$ and $\Gamma\left(\Delta_{c_{1}}\right)=\Delta_{t_{1}}$. Now consider the set $\Delta^{b_{2}}=\left\{\delta_{b_{2}, a_{1}}, \delta_{b_{2}, b_{1}}, \delta_{b_{2}, c_{1}}\right\}$. We have

$$
\begin{aligned}
& \Gamma\left(\delta_{b_{2}, a_{1}}\right) \in \Gamma\left(\Delta^{b_{2}}\right) \cap \Gamma\left(\Delta_{a_{1}}\right)=\Delta^{v_{2}} \cap \Delta_{r_{1}}=\left\{\delta_{v_{2}, r_{1}}\right\} \\
& \Gamma\left(\delta_{b_{2}, b_{1}}\right) \in \Gamma\left(\Delta^{b_{2}}\right) \cap \Gamma\left(\Delta_{b_{1}}\right)=\Delta^{v_{2}} \cap \Delta_{s_{1}}=\left\{\delta_{v_{2}, s_{1}}\right\} \\
& \Gamma\left(\delta_{b_{2}, c_{1}}\right) \in \Gamma\left(\Delta^{v_{2}}\right) \cap \Gamma\left(\Delta_{c_{1}}\right)=\Delta^{v_{2}} \cap \Delta_{t_{1}}=\left\{\delta_{v_{2}, t_{1}}\right\}
\end{aligned}
$$

and so $\Gamma$ is determined on $\Delta^{b_{2}}$. Similarly, we can determine $\Gamma$ on $\Delta^{c_{2}}$ as well. Thus, since every $\delta$ join-irreducible in the six $\Delta$ sets discussed appears in one of $\Delta^{a_{2}}, \Delta^{b_{2}}$, or $\Delta^{c_{2}}$, we have that $\Gamma$ is determined for all of these particular $\delta$ join-irreducibles.

Case 2: Consider automorphisms that map $\Delta^{a_{2}}, \Delta^{b_{2}}, \Delta^{c_{2}}$ to $\Delta_{a_{1}}, \Delta_{b_{1}}, \Delta_{c_{1}}$. We will show, again, that specifying $\Gamma \in \operatorname{Aut}\left(\operatorname{Res}\left(M_{3} \times 2\right)\right)$ on one of $\Delta^{a_{2}}, \Delta^{b_{2}}, \Delta^{c_{2}}$ specifies $\Gamma$ on the remaining five $\Delta$ sets discussed. There are $3 !=6$ ways to map $\Delta^{a_{2}}, \Delta^{b_{2}}, \Delta^{c_{2}}$ to $\left\{\Delta_{a_{1}}, \Delta_{b_{1}}, \Delta_{c_{1}}\right\}$, and then $3 !=6$ ways to specify $\Gamma$ on one of these sets. That makes for a total of $3 !^{2}=36$ automorphisms of $\operatorname{Res}\left(M_{3} \times 2\right)$ that map these upper- $\Delta$ sets to these lower- $\Delta$ sets.

Suppose $\Gamma \in \operatorname{Aut}\left(\operatorname{Res}\left(M_{3} \times 2\right)\right)$ such that $\Gamma\left(\Delta^{a_{2}}\right)=\Delta_{u_{1}}, \Gamma\left(\Delta^{b_{2}}\right)=\Delta_{v_{1}}$, and $\Gamma\left(\Delta^{c_{2}}\right)=\Delta_{w_{1}}$ where $\left\{u_{1}, v_{1}, w_{1}\right\}=\left\{a_{1}, b_{1}, c_{1}\right\}$. Suppose we specify $\Gamma$ on $\Delta^{a_{2}}$ such 
that

$$
\begin{aligned}
& \Gamma\left(\delta_{a_{2}, a_{1}}\right)=\delta_{r_{2}, u_{1}} \\
& \Gamma\left(\delta_{a_{2}, b_{1}}\right)=\delta_{s_{2}, u_{1}} \\
& \Gamma\left(\delta_{a_{2}, c_{1}}\right)=\delta_{t_{2}, u_{1}}
\end{aligned}
$$

where $\left\{r_{2}, s_{2}, t_{2}\right\}=\left\{a_{2}, b_{2}, c_{2}\right\}$.

Note that by Lemma $5.2, \Gamma\left(\delta_{a_{2}, a_{1}}\right)=\delta_{r_{2}, u_{1}}$ implies $\Gamma\left(\Delta_{a_{1}}\right)=\Delta^{r_{2}}$. Similarly, $\Gamma\left(\Delta_{b_{1}}\right)=\Delta^{s_{2}}$ and $\Gamma\left(\Delta_{c_{1}}\right)=\Delta^{t_{2}}$. Now consider the set $\Delta^{b_{2}}=\left\{\delta_{b_{2}, a_{1}}, \delta_{b_{2}, b_{1}}, \delta_{b_{2}, c_{1}}\right\}$ :

$$
\begin{aligned}
& \Gamma\left(\delta_{b_{2}, a_{1}}\right) \in \Gamma\left(\Delta^{b_{2}}\right) \cap \Gamma\left(\Delta_{a_{1}}\right)=\Delta_{v_{1}} \cap \Delta^{r_{2}}=\left\{\delta_{r_{2}, v_{1}}\right\} \\
& \Gamma\left(\delta_{b_{2}, b_{1}}\right) \in \Gamma\left(\Delta^{b_{2}}\right) \cap \Gamma\left(\Delta_{b_{1}}\right)=\Delta_{v_{1}} \cap \Delta^{s_{2}}=\left\{\delta_{s_{2}, v_{1}}\right\} \\
& \Gamma\left(\delta_{b_{2}, c_{1}}\right) \in \Gamma\left(\Delta^{b_{2}}\right) \cap \Gamma\left(\Delta_{c_{1}}\right)=\Delta_{v_{1}} \cap \Delta^{t_{2}}=\left\{\delta_{t_{2}, v_{1}}\right\} .
\end{aligned}
$$

and so $\Gamma$ is determined on $\Delta^{b_{2}}$. Similarly, we can determine $\Gamma$ on $\Delta^{c_{2}}$. Thus, since every $\delta$ join-irreducible in the six $\Delta$ sets discussed appears in one of $\Delta^{a_{2}}, \Delta^{b_{2}}$, or $\Delta^{c_{2}}$, we have that $\Gamma$ is determined for all of these particular $\delta$ join-irreducibles.

Combining Case 1 and Case 2, we have that there are $36+36=72$ ways for an order automorphisms of $\operatorname{Res}\left(M_{3} \times 2\right)$ to map the sets $\Delta^{a_{2}}, \Delta^{b_{2}}, \Delta^{c_{2}}, \Delta_{a_{1}}, \Delta_{b_{1}}, \Delta_{c_{1}}$.

LEMMA 5.4. Suppose $\Gamma \in \operatorname{Aut}\left(\operatorname{Res}\left(M_{3} \times 2\right)\right)$ is known on $\Delta^{a_{2}}, \Delta^{b_{2}}, \Delta^{c_{2}}, \Delta_{a_{1}}, \Delta_{b_{1}}$, and $\Delta_{c_{1}}$, and assume the image of any $\delta$ map under $\Gamma$ is also a $\delta$ map. Then this is enough to determine $\Gamma$ on $\Delta^{x}$ and $\Delta_{y}$.

Proof. We will discuss two separate, but very similar cases.

Case 1: Suppose uppers $\leftrightarrow$ uppers and lowers $\leftrightarrow$ lowers. By this, we mean that for any $u_{2} \in\left\{a_{2}, b_{2}, c_{2}\right\}$, there exists $v_{2}$ in $\left\{a_{2}, b_{2}, c_{2}\right\}$ such that $\Gamma\left(\Delta^{u_{2}}\right)=\Delta^{v_{2}}$ and for any $r_{1} \in\left\{a_{1}, b_{1}, c_{1}\right\}$, there exists $s_{1} \in\left\{a_{1}, b_{1}, c_{1}\right\}$ such that $\Gamma\left(\Delta_{r_{1}}\right)=\Delta_{s_{1}}$.

Now, let $u_{2}, v_{2}, r_{1}, s_{1}$ be as described above. Since $\Gamma\left(\Delta^{u_{2}}\right)=\Delta^{v_{2}}$, we know that 
$\Gamma\left(\bigvee\left\{\delta \mid \delta \in \Delta^{u_{2}}\right\}\right)=\Gamma\left(\bigvee\left\{\delta \mid \delta \in \Delta^{v_{2}}\right\}\right)$, and thus $\Gamma\left(\delta_{u_{2}, x}\right)=\delta_{v_{2}, x}$. Notice that the only $\delta$ map that covers $\delta_{u_{2}, x}$ is $\delta_{u_{2}, 1}$ and the only $\delta$ map that covers $\delta_{v_{2}, x}$ is $\delta_{v_{2}, 1}$. So we can conclude, since $\delta \mathrm{s}$ map to $\delta \mathrm{s}$, that $\Gamma\left(\delta_{u_{2}, 1}\right)=\delta_{v_{2}, 1}$. Now, consider the $\delta$ join-irreducibles in $\downarrow \delta_{u_{2}, 1}$; they include those in $\Delta^{u_{2}}$ and $\delta_{u_{2}, y}$. Similarly, the $\delta$ joinirreducibles in $\downarrow \delta_{v_{2}, 1}$ include $\Delta^{v_{2}}$ and $\delta_{v_{2}, y}$. Since we already know $\Gamma\left(\Delta^{u_{2}}\right)=\Delta^{v_{2}}$, we are left to conclude that $\delta_{u_{2}, y}=\delta_{v_{2}, y}$. Because $u_{2}$ and $v_{2}$ were general elements of $\left\{a_{2}, b_{2}, c_{2}\right\}$, we now conclude that $\Delta_{y}$ is determined.

Similarly, since $\Gamma\left(\Delta_{r_{1}}\right)=\Delta_{s_{1}}$, we know that $\Gamma\left(\bigvee\left\{\delta \mid \delta \in \Delta_{r_{1}}\right\}\right)=\Gamma(\bigvee\{\delta \mid$ $\left.\left.\delta \in \Delta_{s_{1}}\right\}\right)$, and thus $\Gamma\left(\delta_{y, r_{1}}\right)=\delta_{y, s_{1}}$. Now the only $\delta$ map that covers $\delta_{y, r_{1}}$ is $\delta_{0, r_{1}}$ and, similarly, the only $\delta$ map that covers $\delta_{y, s_{1}}$ is $\delta_{0, s_{1}}$. Since $\delta$ s map to $\delta$ s, we conclude that $\Gamma\left(\delta_{0, r_{1}}\right)=\delta_{0, s_{1}}$. Now, consider the $\delta$ join-irreducibles in $\downarrow \delta_{0, r_{1}}$; they include $\Delta_{r_{1}}$ and $\delta_{x, r_{1}}$. Similarly, the $\delta$ join-irreducibles in $\downarrow \delta_{0, s_{1}}$ include $\Delta_{s_{1}}$ and $\delta_{x, s_{1}}$. Since we already know $\Gamma\left(\Delta_{r_{1}}\right)=\Delta_{s_{1}}$, we are left to conclude that $\Gamma\left(\delta_{x, r_{1}}\right)=\delta_{x, s_{1}}$. Because $r_{1}$ and $s_{1}$ were general elements of $\left\{a_{1}, b_{1}, c_{1}\right\}$, we can now conclude that $\Delta^{x}$ is determined.

Case 2: Suppose uppers $\leftrightarrow$ lowers. By this, we mean that for any $u_{2} \in$ $\left\{a_{2}, b_{2}, c_{2}\right\}$, there exists $v_{1}$ in $\left\{a_{1}, b_{1}, c_{1}\right\}$ such that $\Gamma\left(\Delta^{u_{2}}\right)=\Delta_{v_{1}}$ and for any $r_{1} \in$ $\left\{a_{1}, b_{1}, c_{1}\right\}$, there exists $s_{2} \in\left\{a_{2}, b_{2}, c_{2}\right\}$ such that $\Gamma\left(\Delta_{r_{1}}\right)=\Delta^{s_{2}}$.

Let $u_{2}, v_{1}, r_{1}, s_{2}$ be described as above. Since $\Gamma\left(\Delta^{u_{2}}\right)=\Delta_{v_{1}}$, we know that $\Gamma\left(\bigvee\left\{\delta \mid \delta \in \Delta^{u_{2}}\right\}\right)=\Gamma\left(\bigvee\left\{\delta \mid \delta \in \Delta_{v_{1}}\right\}\right)$, and thus $\Gamma\left(\delta_{u_{2}, x}\right)=\delta_{y, b_{1}}$. The only $\delta$ map that covers $\delta_{u_{2}, x}$ is $\delta_{u_{2}, 1}$ and the only $\delta$ map that covers $\delta_{y, b_{1}}$ is $\delta_{0, v_{1}}$. Since $\delta \mathrm{s}$ map to $\delta \mathrm{s}$, we conclude that $\Gamma\left(\delta_{u_{2}, 1}\right)=\delta_{0, v_{1}}$. Now, consider the $\delta$ join-irreducibles in $\downarrow \delta_{u_{2}, 1}$; they include $\Delta^{u_{2}}$ and $\delta_{u_{2}, y}$. Similarly, the $\delta$ join-irreducibles in $\downarrow \delta_{0, v_{1}}$ include $\Delta_{v_{1}}$ and $\delta_{x, v_{1}}$. Since we already know $\Gamma\left(\Delta^{u_{2}}\right)=\Delta_{v_{1}}$, we are left to conclude that $\Gamma\left(\delta_{u_{2}, y}\right)=\delta_{x, v_{1}}$. Since $u_{2}$ and $v_{1}$ were general elements, we can now conclude that $\Delta_{y}$ is determined.

Similarly, since $\Gamma\left(\Delta_{r_{1}}\right)=\Delta^{s_{2}}$, we know that $\Gamma\left(\bigvee\left\{\delta \mid \delta \in \Delta_{r_{1}}\right\}\right)=\Gamma(\bigvee\{\delta \mid$ 
$\left.\delta \in \Delta^{s_{2}}\right\}$ ), and thus $\Gamma\left(\delta_{y, r_{1}}\right)=\delta_{s_{2}, x}$. Now the only $\delta$ map that covers $\delta_{y, r_{1}}$ is $\delta_{0, r_{1}}$ and, similarly, the only $\delta$ map that covers $\delta_{s_{2}, x}$ is $\delta_{s_{2}, 1}$. Since $\delta$ s map to $\delta$ s, we conclude that $\Gamma\left(\delta_{0, r_{1}}\right)=\delta_{s_{2}, 1}$. Now, consider the $\delta$ join-irreducibles in $\downarrow \delta_{0, r_{1}}$; they include $\Delta_{r_{1}}$ and $\delta_{x, r_{1}}$. Similarly, the $\delta$ join-irreducibles in $\downarrow \delta_{s_{2}, 1}$ include $\Delta^{s_{2}}$ and $\delta_{s_{2}, y}$. Since we already know $\Gamma\left(\Delta_{r_{1}}\right)=\Delta^{s_{2}}$, we are left to conclude that $\Gamma\left(\delta_{x, r_{1}}\right)=\delta_{s_{2}, y}$. Because $r_{1}$ and $s_{2}$ were general elements, we can now conclude that $\Delta^{x}$ is determined.

Note that Lemma 5.4 is the only result in this chapter that depends on the assumption that $\delta$ functions map to $\delta$ functions. Therefore, if we can find a way to prove this result without this assumption, we will have proved that every order automorphism of $\operatorname{Res}\left(M_{3} \times 2\right)$ is induced.

LEMMA 5.5. If $\Gamma \in \operatorname{Aut}\left(\operatorname{Res}\left(M_{3} \times 2\right)\right)$ is known on the $\delta$ join-irreducible elements, then it is fully determined on all of $\operatorname{Res}\left(M_{3} \times 2\right)$.

Proof. We need only show that $\Gamma$ being known on the $\delta$ join-irreducible elements is enough to determine $\Gamma$ on the remaining join-irreducible elements, the permutations. Once $\Gamma$ is known on all of the join-irreducibles, it is known on all of $\operatorname{Res}\left(M_{3} \times 2\right)$, since every element is the join of join-irreducibles.

Let $\left\{u_{1}, v_{1}, w_{1}\right\}=\left\{r_{1}, s_{1}, t_{1}\right\}=\left\{a_{1}, b_{1}, c_{1}\right\}$. Suppose $p \in \operatorname{Res}\left(M_{3} \times 2\right)$ is the permutation join-irreducible element defined by

$$
\begin{array}{ll}
p\left(u_{1}\right)=r_{1} & p\left(v_{1}\right)=s_{1} \\
p\left(w_{1}\right)=t_{1} & p(y)=0 .
\end{array}
$$

Then there exists a cover $c$ of $p$ in $\operatorname{Res}\left(M_{3} \times 2\right)$ defined by

$$
\begin{array}{ll}
c\left(u_{1}\right)=x & c\left(v_{1}\right)=s_{1} \\
c\left(w_{1}\right)=t_{1} & c(y)=0
\end{array}
$$

Consider the principal downset of this cover: $\downarrow c=\left\{c, \delta_{v_{2}, t_{1}}, \delta_{w_{2}, s_{1}}, p, \overline{0}\right\}$. Notice there are three join-irreducibles in the downset: two $\delta$ join-irreducibles and the 
permutation join-irreducible $p$. These three elements together form a set with the " $M_{3}$ property" previously discussed, and this is enough to show $p$ is determined. To assure the reader, notice that $\Gamma(c)=\Gamma\left(\delta_{v_{2}, t_{1}}\right) \vee \Gamma\left(\delta_{w_{2}, s_{1}}\right)$. Since $\Gamma$ is known on the $\delta$ join-irreducibles, and because of the behavior of $\Gamma$ on these $\delta$ s discussed in Lemmas 5.1, 5.2 and 5.3, we can conclude that $\Gamma(c)=c^{\prime}$ where $c^{\prime}$ is of the same form as $c$. There is only one permutation join-irreducible, call it $p^{\prime}$, below $c^{\prime}$, and so we can conclude $\Gamma(c)=c^{\prime} \Longrightarrow \Gamma(p)=p^{\prime}$. Since $p$ was a general permutation joinirreducible, we have shown that knowing $\Gamma$ on the $\delta$ join-irreducibles determines $\Gamma$ on the remaining join-irreducibles, and thus all of $\operatorname{Res}\left(M_{3} \times 2\right)$.

THEOREM 5.1. Suppose $\Gamma \in \operatorname{Aut}\left(\operatorname{Res}\left(M_{3} \times 2\right)\right)$ and $\Gamma$ sends $\delta$ maps to $\delta$ maps. If $f \in \operatorname{Res}\left(M_{3} \times 2\right)$ with $f^{+}$its residual, then $\Gamma$ is of one of the following forms:

(i) $\Gamma(f)=\theta \circ f \circ \gamma$ where $\theta, \gamma \in \operatorname{Aut}\left(M_{3} \times 2\right)$;

(ii) $\Gamma(f)=\alpha \circ f^{+} \circ \beta$ where $\alpha, \beta$ are dual isomorphisms of $M_{3} \times 2$.

Proof. This is merely a counting argument. We have shown that $\Gamma$ must keep the sets $\Delta^{a_{2}}, \Delta^{b_{2}}, \Delta^{c_{2}}, \Delta_{a_{1}}, \Delta_{b_{1}}, \Delta_{c_{1}}$ invariant, and Lemma 5.3 tells us there are 72 ways $\Gamma$ can do this. Lemmas 5.4 and 5.5 ensure us that, since $\Gamma$ maps $\delta$ s to $\delta$ s, knowing $\Gamma$ on these six $\Delta$ sets determines $\Gamma$ on all of $\operatorname{Res}\left(M_{3} \times 2\right)$. So, there are 72 order automorphisms of $\operatorname{Res}\left(M_{3} \times 2\right)$ that map $\delta$ s to $\delta$ s.

We can also count the induced order automorphisms described by this theorem. The only order automorphisms of $M_{3} \times 2$ are those that permute $a_{1}, b_{1}, c_{1}$, so there are 6 choices for $\theta$ and $\gamma$ and thus 36 type (i) order automorphisms of $\operatorname{Res}\left(M_{3} \times 2\right)$. The only dual isomorphisms of $M_{3} \times 2$ are characterized by permuting $a_{2}, b_{2}, c_{2}$, so there are 6 choices for $\alpha$ and $\beta$ and thus 36 type (ii) order automorphisms of $\operatorname{Res}\left(M_{3} \times 2\right)$. So there are 72 induced order automorphisms. Thus, every order automorphism of $\operatorname{Res}\left(M_{3} \times 2\right)$ that sends $\delta$ s to $\delta$ s is induced. 
The critical part of our statement here is the assumption that an order automorphism of $\operatorname{Res}\left(M_{3} \times 2\right)$ maps $\delta \mathrm{s}$ to $\delta \mathrm{s}$. This is a strong assumption, and frankly the writer's intuition about its validity changes often. We have been able to rephrase the question in several complicated ways while looking at the elements and structure of $\operatorname{Res}\left(M_{3} \times 2\right)$, but the rephrasings have not led to any clearer of a picture. We have an idea regarding how an order automorphism that does not send $\delta$ s to $\delta$ s (if it exists) would map the low elements in the lattice $\operatorname{Res}\left(M_{3} \times 2\right)$. However, other than taking on a large computer programming project, we cannot find a way to test if such a function would truly be an order automorphism. The lattice $\operatorname{Res}\left(M_{3} \times 2\right)$ is just too large and complicated of a structure. We will discuss our future plans to gain clarity on this problem in the following conclusion. 


\section{CHAPTER 6 REMARKS AND CONCLUSION}

When setting out to investigate the order automorphisms of $\operatorname{Res}(L)$ where $L$ is finite and nondistributive, our intuition was that not many order automorphisms would be induced. This was because we knew of examples in the distributive case where there exist non-induced order automorphisms, and typically you lose elegant results when considering distributive versus nondistributive lattices. It came as a surprise, then, when we showed that order automorphisms of the residuated maps of $M_{3}$ and $N_{5}$, the two canonical nondistributive lattices, are indeed all induced. Our intuition for general nondistributive lattice $L$ is still that there exist cases with non-induced order automorphisms, so it is our goal now to find an example of such a lattice. This was our hope with $M_{3} \times 2$, but the methods we have used so far to attack the problem have not been strong enough.

Thus, our next goal is to look at the bigger, more abstract picture. What characteristics of $L$ give us insight into characteristics of $\operatorname{Res}(L)$ ? For instance, we know from Shmuely [25] that $\operatorname{Res}(L)$ is distributive if and only if $L$ is distributive. We have shown by example that the same is not true for modularity. A lattice $L$ being modular does not necessarily ensure $\operatorname{Re} s(L)$ is modular, the lattice $M_{4}$ being a counterexample.

Next we would like to investigate the relationship between $\operatorname{Res}(L \times M)$, $\operatorname{Res}(L)$, and $\operatorname{Res}(M)$. Farley [10] has some results on the partially ordered set of non-decreasing maps, so perhaps we can extend or manipulate his results to the subset of residuated maps. We investigated some of the possibilities here when 
we noticed that there are $2500=50^{2}$ elements in $\operatorname{Res}\left(M_{3} \times 2\right)$, while there are 50 elements in $\operatorname{Res}\left(M_{3}\right)$. However, we found that $\operatorname{Res}\left(M_{3} \times 2\right) \nsucceq \operatorname{Res}\left(M_{3}\right) \times$ $\operatorname{Res}\left(M_{3}\right)$ because the number of join-irreducibles in each is different. Finding a link between $\operatorname{Res}(L \times M)$, Res $(L)$, and $\operatorname{Res}(M)$, however, may prove fruitful to finding an example of a lattice for which not all the order automorphisms are induced, as such a product forced this is in the distributive case. For instance, we may look next at the residuated maps on $M_{3} \times M_{3}$ or on $M_{3} \times M_{2} \cong M_{3} \times 2 \times 2$ because a product of two identical lattices forced non-induced order automorphisms in the distributive case.

We would also like to investigate the relationship between $\operatorname{Res}(K)$ and $\operatorname{Res}(L)$ when $K$ is a sublattice of $L$. Since a nondistributive lattice is characterized by having one or both of $M_{3}$ or $N_{5}$ as a sublattice, such results combined with the results of this dissertation could lead to a larger, more powerful result for all nondistributive lattices.

We hope by gearing our investigation in a new, more abstract direction, we can get more definitive results. However, residuated maps and the lattice of residuated maps on nondistributive lattices have proven to be complex objects without many nice characteristics. The challenge will be to break through the complexity and find the most general results we can, which then we can hopefully apply to more specific cases. 


\section{REFERENCES}

[1] R. Belohlavek and P. Osicka, Triadic fuzzy Galois connections as ordinary connections, Fuzzy Sets and Systems 249 (2014), 83-99.

[2] G. Birkhoff, Lattice Theory, Pergamon Press, Oxford, 1972.

[3] T.S. Blyth and M.F. Janowitz, Residuation Theory, Pergamon Press, Oxford, 1972.

[4] Cristofor L. Cristofor, D. and D. Simovici, Galois connections and data mining, J. of Universal Comp. Sci. 6 (2000), no. 1, 60-73.

[5] B.A. Davey and H.A. Priestley, Introduction to Lattices and Order, 2 ed., Cambridge University Press, Cambridge, 2002.

[6] F. Domenach and B. Leclerc, On the roles of galois connections in classification, Exploratory Data Analysis in Empirical Research, Studies in Classification, Data Analysis, and Knowledge Organization, Springer Berlin Heidelberg, 2003, pp. 31-40.

[7] D. Duffus, Automorphisms and products of ordered sets, Algebra Universalis 19 (1984), no. 3, 366-369.

[8]

[9] Jónsson B. Duffus, D. and I. Rival, Structure results for function lattices, Canad. J. Math. 30 (1978), no. 2, 392-400. 
[10] J.D. Farley, The automorphism group of a function lattice: a problem of Jónsson and McKenzie, Algebra Universalis 36 (1996), no. 1, 8-45.

[11] _ Solution to problems of Schmidt and Quackenbush from 1974 and 1985: tensor products of semilattices, Mathematica Pannonica 22 (2011), 135-147.

[12] The OEIS Foundation Inc., The on-line encyclopedia of integer sequences.

[13] M.F. Janowitz, Cluster analysis based on posets, Selected Contributions in Data Analysis and Classification, Studies in Classification, Data Analysis, and Knowledge Organization, Springer Berlin Heidelberg, 2007, pp. 213-223.

[14] _ Ordinal and Relational Clustering, Interdiscplinary Mathematical Sciences, vol. 10, World Scientific, New Jersey, 2010.

[15] M.F. Janowitz and B. Schweizer, Ordinal and percentile clustering, Mathematical Social Sciences 18 (1989), no. 2, 135-186.

[16] B. Jónsson and R. McKenzie, Powers of partially ordered sets: cancellation and refinement properties, Math. Scand. 51 (1982), no. 1, 87-120.

[17] _ Powers of partially ordered sets: the automorphism group, Math. Scand. 51 (1982), no. 1, 121-141.

[18] Marshall Lagani, personal communication.

[19] A. Melton, D.A. Schmidt, and G.E. Strecker, Category Theory and Computer Programming, ch. Galois connections and computer science applications, pp. 299-312, Springer, Berlin, 1986.

[20] R.C. Powers, Order automorphisms of spaces of nondecreasing functions, Math. Anal. Appl. 136 (1988), 112-123.

[21] _ Induced residuated maps, Order 7 (1990), 83-96. 
[22] G.N. Raney, Tight galois connections and complete distributivity, Transactions of the AMS 97 (1960), no. 3, 418-426.

[23] E.A. Schreiner, Tight residuated mappings, In: Proceedings of the University of Houston, Lattice Theory Conference (Houston, Texas 1973), 519-530.

[24] B. Schweizer and A. Sklar, Probabilistic Metric Spaces, North-Holland, Amsterdam, 1983.

[25] Z. Shmuely, The structure of galois connections, Pacific J. of Math. 54 (1974), no. 2, 209-225.

[26] R. Wille, Cancellation and refinement results for function lattices, Houston J. Math. 6 (1980), no. 3, 431-437. 


\title{
CURRICULUM VITAE
}

\author{
Erika D. Foreman
}

\section{EDUCATION}

University of Louisville, Louisville, $K Y$

Ph.D. in Applied and Industrial Mathematics Expected August 2015 Areas of Concentration: Lattice Theory and Partially Ordered Sets

Title of Dissertation: "Order Automorphisms on the Lattice of Residuated Maps of Some Special Nondistributive Latices.

Advisor: Dr. Thomas Riedel

University of Louisville, Louisville, $K Y$

M.A. Mathematics

May 2011

Georgetown College, Georgetown, KY

B.S. Mathematics and Computer Science

May 2009

Double major in Mathematics and Computer Science, minor in Physics. Graduated Summa Cum Laude.

\section{TEACHING EXPERIENCE}

University of Louisville, Louisville, $K Y$

Graduate Teaching Assistant

August 2010-Present

Primary Instructor for: MATH 151- Math for Elementary Education I; MATH 190Precalculus; MATH 205- Calculus I.

Teaching Assistant for: MATH 105- Contemporary Mathematics; MATH 107- Finite Mathematics, MATH 111- College Algebra.

University of Louisville, Louisville, $K Y$

GEMS Fellow

August 2009-June 2010

The Groundwork Education in Mathematics and Science (GEMS) project was a collaborative effort between the University of Louisville and the Jefferson County Public Schools in Louisville, KY. As a GEMS Fellow, I was teamed with a JCPS sixth grade math teacher. Together we developed, planned, and implemented inquirybased math lessons. Approximately 10-15 hours per week were spent on instruction

in the classroom, plus an additional 3-6 hours per week were spent in seminars 
and completing assignments about the effectiveness and implementation of inquirybased learning in the middle-grades mathematics curriculum.

\section{RELATED EXPERIENCE}

Department of Mathematics, University of Louisville, Louisville, KY

Dual Credit Coordinator

August 2012-Present

Coordinate dual credit courses between the University of Louisville and participating high schools in and around Louisville, KY. Verify teacher qualifications, verify student eligibility, assist in student enrollment, oversee course content and textbook selection, oversee assessment and grading of the courses, evaluate dual credit instructors; wrote Dual Credit Mathematics Handbook.

Private Tutor, Louisville, KY

Tutor

2011-Present

Tutor middle school, high school, and undergraduate students in Algebra, Geometry, Precalculus, and Calculus I.

YUM Brands, Louisville, $K Y$

Computer Programming Intern

Summer 2008, Summer 2009 Interned in the Financial department, writing SQR scripts and SQL queries to collect needed data from invoices. Completed a project to enable small vendors to send invoices over SFTP.

Department of Mathematics, Computer Science, and Physics, Georgetown College, Georgetown, $K Y$

Undergraduate Assistant

August 2007- May 2009

Assisted computer science professors in grading; tutored both Business Calculus and Intro. to Computer Science; lectured a 7-session Trigonometry review for Calculus I students; oversaw the student computer lab.

The Writing Center, Georgetown College, Georgetown, KY

Writing Tutor

August 2007- May 2009

Reviewed structure, grammar, and content of written assignments one-on-one with students.

\section{PAPERS AND PUBLICATIONS}

Erika D. Foreman, "Order Automorphisms of Residuated Mappings Between Certain Nondistributive Lattices," submitted.

\section{PRESENTATIONS}

"An investigation of some lattice theoretic properties of $\operatorname{Res}\left(M_{3}\right)$," 2014 Annual KYMAA Meeting, Murray State University, Murray, KY, March 28, 2014. 
"Order Automorphisms of Function Lattices," Special Session on Partially Ordered Sets, AMS Southeastern Sectional Meeting, University of Louisville, Louisville, KY, October 5, 2013.

"Order Automorphisms on the Lattice of Residuated Mappings of $M_{n}$," Candidacy Examination, University of Louisville, Louisville, KY, May 7, 2013.

"Counts of Residuated Maps on Two Interesting Lattices," 2013 Annual KYMAA Meeting, Transylvania University, Lexington, KY, April 5, 2013.

"Order Automorphisms of Functions Lattices," Algebra Seminar, University of Louisville, Louisville, KY, March 22, 2013.

\section{AWARDS}

Ken F. and Sandra S. Hohman Graduate Fellowship In Mathematics, University of Louisville

2012

Graduate Teaching Assistantship, University of Louisville

August 2010

GEMS Fellowship, University of Louisville

August 2009

Phi Kappa Phi

2008

Beth Awards for Computer Science, Math, \& Physics, Georgetown College 2008

Most Promising Computer Science Student Award, Georgetown College 2007

Freshman Writing Award, Georgetown College

2006

Dean's List, each semester, Georgetown College

2005-2009 